\title{
Synthesis of inositol glycan cyclic phosphates
}

\author{
Christine H. Jaworek, Sarah Iacobucci, Pericles Calias, Marc d'Alarcao* \\ Michael Chemistry Laboratory, Department of Chemistry, Tufts University, Medford, MA 02155, USA \\ Received 7 November 2000; accepted 26 January 2001
}

\begin{abstract}
An efficient synthesis of tri-, tetra-, and pentasaccharide cyclic phosphates 1-5, structurally related to natural inositol phosphate glycans, is reported. The title compounds were assembled by PhSeOTf-promoted glycosylation of the known glucosamine precursor, $t$-butyldimethylsilyl 2-azido-3,6-di- $O$-benzyl-2-deoxy- $\beta$-D-glucopyranoside (8) with protected 1-methylthio mono-, di-, and trimannosides $7 \mathbf{a}-\mathbf{c}$, and, after conversion into glycosyl fluorides, $\mathrm{Cp}_{2} \mathrm{ZrCl}_{2}-$ AgOTf-promoted glycosylation of differentially protected optically pure 1D-myo-inositol 11. The syntheses were completed by installing the cyclic phosphate moieties with methylpyridinium dichlorophosphate and finally, removal of all protecting groups by dissolving-metal reduction. (C) 2001 Elsevier Science Ltd. All rights reserved.
\end{abstract}

Keywords: Inositol phosphate glycans; Cyclic phosphates; 1D-myo-inositol derivatives

\section{Introduction}

In the US, approximately 16 million people suffer from diabetes mellitus. Ninety to ninety-five percent of these are afflicted by non-insulin dependent diabetes mellitus (NIDDM), a condition characterized by insulin resistance. Diminished binding of insulin to its receptor usually does not adequately account for the decreased responsiveness on the cellular level. Therefore, NIDDM may be considered a disease of insulin signal transduction. ${ }^{1}$

During the past 15 years, small inositol-containing oligosaccharides have been implicated as signaling molecules in the insulin signal transduction pathway. ${ }^{2}$ These oligosaccharides are believed to be structurally similar to the glycosylphosphatidyl-inositol (GPI) membrane anchors. This idea is supported by the observation that the compound obtained by

\footnotetext{
* Corresponding author. Fax: + 1-617-6273443.

E-mail address: mdalarca@tufts.edu (M. d'Alarcao).
}

PI-PLC and Pronase release of the variant surface glycoprotein (VSG) GPI anchor from variant clone 118 of Trypanosoma bruceii strain 427 , presumed to have structure $\mathbf{6}$, is insulin mimetic. ${ }^{3,14}$

However, due to the miniscule amounts of biological material available and the heterogeneity of many isolates, the precise chemical structures of these putative insulin mediators are not known. At least two general classes of compounds have been identified. One class, referred to by Larner's group ${ }^{4}$ as the $\mathrm{pH} 1.3$ mediator because of the $\mathrm{pH}$ of an eluting solvent during purification, inhibits cAMP-dependent protein kinase and contains myoinositol and glucosamine. ${ }^{5}$ The other, so-called $\mathrm{pH} 2$ mediator, stimulates pyruvate dehydrogenase phosphatase and contains chiro-inositol and galactosamine. ${ }^{6}$

The lack of complete structural characterization has led to intense synthetic efforts by several groups ${ }^{7-11}$ in the hope of determining the structural features necessary for mimicking insulin action. Herein, we report the syn- 


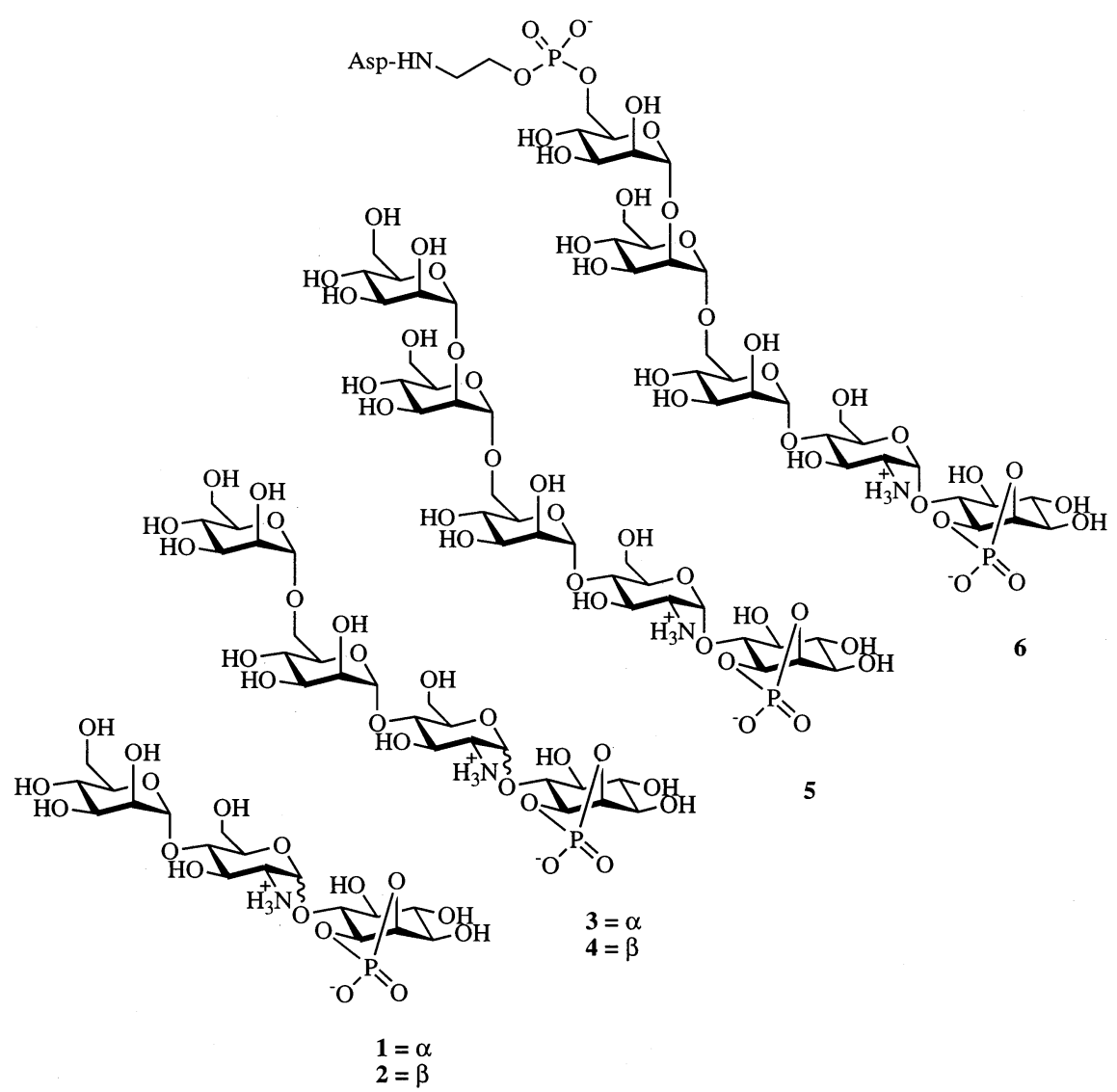

Fig. 1. Synthetic compounds $\mathbf{1 - 5}$, and natural VSG membrane anchor fragment $\mathbf{6}$.

thesis of oligosaccharides $\mathbf{1}-\mathbf{5} .^{12,13 \dagger}$ Compounds $\mathbf{1}, \mathbf{3}$, and $\mathbf{5}$ constitute the terminal tri-, tetra-, and pentasaccharide portion of $\mathbf{6}$, the presumed structure of the VSG anchor fragment from T. bruceii variant 118. Compounds $\mathbf{2}$ and $\mathbf{4}$ differ from the truncated VSG anchor in that there is a $\beta$ linkage between the inositol and the glucosamine residues (Fig. 1).

\section{Results and discussion}

Synthetic strategy. - The syntheses of compounds 1-5 were designed to be modular so that assembly of a variety of oligosaccharides could be achieved rapidly (Scheme 1). The two terminal portions of each target compound, an oligomannose unit and an inositol unit,

\footnotetext{
${ }^{\dagger}$ Our synthesis of $\mathbf{1}$ has been communicated previously, without experimental details (Ref. 11) and has been synthesized independently (Ref. 10).

\# A report on the synthesis of $\mathbf{5}$ by a different procedure appeared at the time of submission of this manuscript. ${ }^{13}$
}

would be attached to a suitably protected linking glucosamine unit. To minimize the number of manipulations, all protecting groups remaining after final assembly of the oligosaccharides were chosen to be removable in a single deprotection step.

The known glucosamine precursor $t$ butyldimethylsilyl 2-azido-3,6-di- $O$-benzyl-2deoxy- $\beta$-D-glucopyranoside $(\mathbf{8})^{15}$ satisfied these criteria. We envisioned glycosylating the 4-position of $\mathbf{8}$ with mono-, di-, and trimannoses $7 \mathbf{a},{ }^{16} \mathbf{7 b}$, or $7 \mathbf{c}$ to provide the corresponding di-, tri-, and tetrasaccharides $\mathbf{9 a}-\mathbf{c}$. The methyl 1-thio- $\alpha$-glycosides $7 \mathbf{a}-\mathbf{c}$ were selected to utilize Ogawa's $\alpha$-selective phenylselenyl triflate-promoted glycosylation procedure. ${ }^{22}$ After desilylation and conversion into the glycosyl fluorides $10 a-c$, zirconocene dichloridepromoted coupling ${ }^{17}$ to myo-inositol 11 would produce fully protected tri-, tetra-, and pentasaccharides 12a-c, respectively. This glycosylation method was chosen since both the $\alpha$ and $\beta$ anomers of the oligosaccharides were desired. Recent work by Müller's group ${ }^{8}$ has 
demonstrated that some IPGs with $\beta$-D inositol linkages are insulin mimetic.

Inositol 11 was chosen to allow selective removal of the carbonate moiety of 12a-c, thereby freeing the hydroxyl groups to be esterified in the cyclic phosphate moiety of the targets. Cyclic phosphorylation could then be accomplished in one step by addition of methylpyridinium dichlorophosphate (31). ${ }^{18}$ Finally, removal of the benzyl protecting groups and reduction of the azido functionality in a single reduc- tive deprotection step would produce targets 1-5.

Synthesis of mannose methyl 1-thioglycosides 7a-c.-Selective dimethoxytritylation of the C-6 hydroxyl of the known mannose glycoside $\mathbf{1 3}^{19,20}$ (Scheme 2) with 4,4'-dimethoxytrityl tetrafluoroborate ${ }^{21}$ yielded triol 14. Exhaustive benzylation under standard conditions (i) $\mathrm{NaH}, \mathrm{DMF}, 0{ }^{\circ} \mathrm{C}$; (ii) $\mathrm{BnBr}$ ) yielded fully protected 15. Removal of the DMT protecting group with 50\% HOAc provided alcohol 16 in $64 \%$ overall yield from 13 .
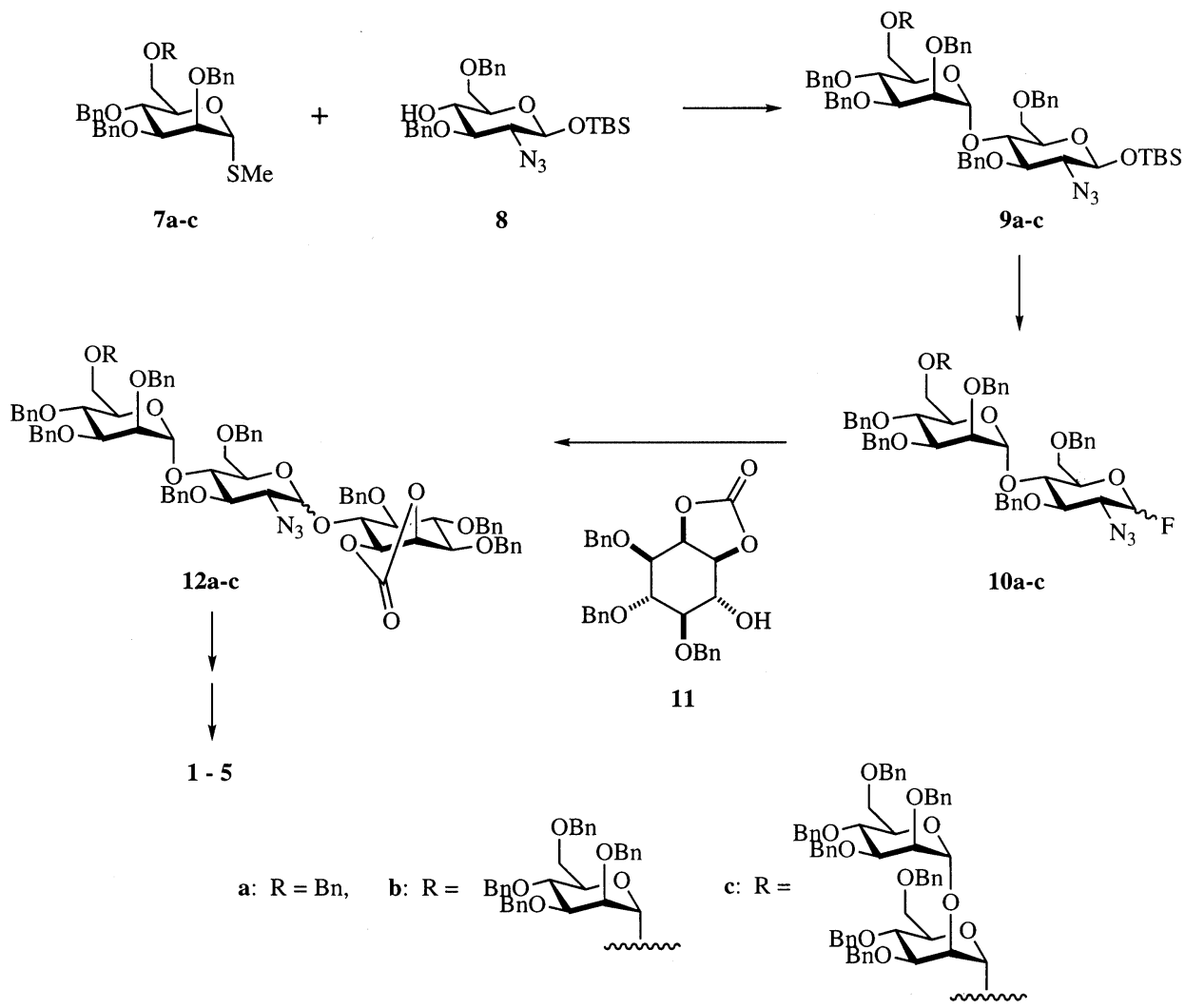

Scheme 1. Overview of the syntheses of compounds $\mathbf{1}-\mathbf{5}$.

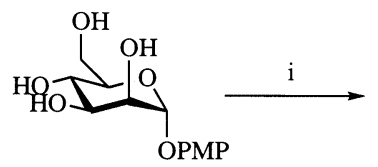

13

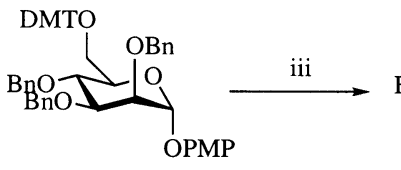

15

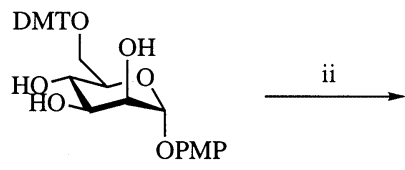

14
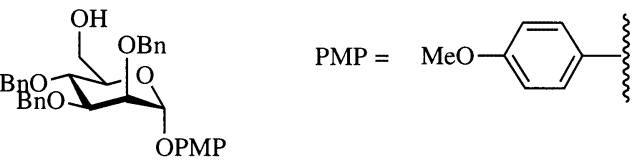

16

Scheme 2. (i) DMTBF, DBMP, $\mathrm{CH}_{3} \mathrm{CN}$, reflux (92\%); (ii) (a) $\mathrm{NaH}, \mathrm{DMF}$; (b) $\mathrm{BnBr}$; (iii) $\mathrm{CHCl}_{3}, 50 \% \mathrm{CH}_{3} \mathrm{CO}_{2} \mathrm{H}, 80{ }^{\circ} \mathrm{C}$ (70\%, from 14). 


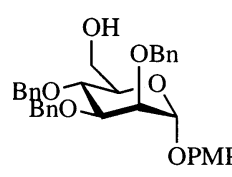

16

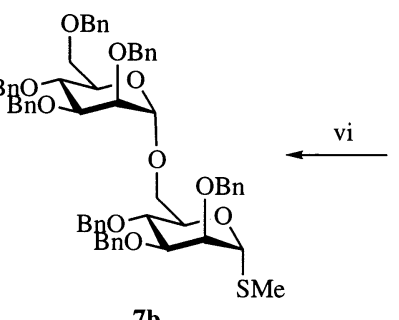

$7 \mathbf{b}$

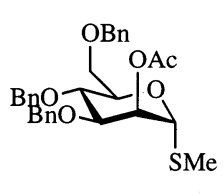

17

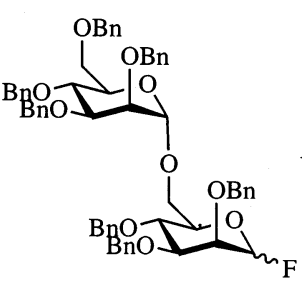

22

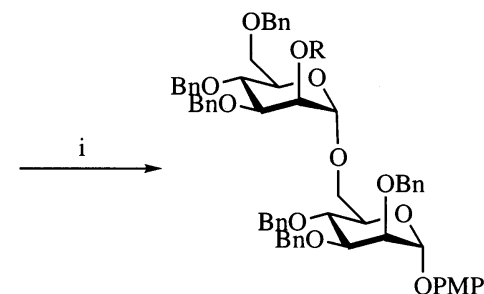

ii $18: \mathrm{R}=\mathrm{Ac}$ ii $\begin{cases}\text { 19: } & R=H \\ \text { 20: } & R=B n\end{cases}$ iv

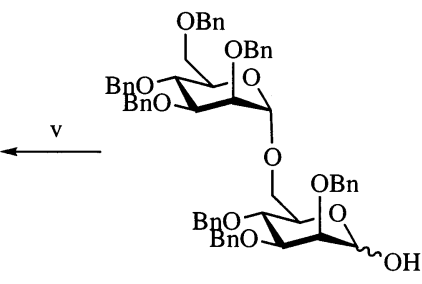

21

Scheme 3. (i) $\mathrm{PhSeCl}, \mathrm{AgOTf}$, toluene, $4 \AA$ molecular sieves, $-40^{\circ} \mathrm{C}(56-71 \%)$; (ii) $\mathrm{NH}_{3}, \mathrm{MeOH}, 20{ }^{\circ} \mathrm{C}(78 \%)$; (iii) (a) $\mathrm{NaH}$, DMF; (b) $\mathrm{BnBr}\left(85 \%\right.$ ); (iv) $\mathrm{CH}_{3} \mathrm{CN}-\mathrm{H}_{2} \mathrm{O}$, + $1.55 \mathrm{~V}, 0.1 \mathrm{M} \mathrm{Bu}_{4} \mathrm{NPF}_{6}, 20{ }^{\circ} \mathrm{C}$ (74\%); (v) DAST, THF (86\%); (vi) Bu 3 SnSMe, $\mathrm{SnCl}_{4}, \mathrm{ClCH}_{2} \mathrm{CH}_{2} \mathrm{Cl}(85 \%)$.

Coupling of 16 to the known methyl 1thiomannoside $17^{16}$ (Scheme 3) using Ogawa's protocol $^{22}$ yielded the differentially protected mannose disaccharide 18. The $\alpha$ configuration of the glycosidic linkage in the product was confirmed by the ${ }^{13} \mathrm{C}-{ }^{1} \mathrm{H}$ coupling constant $(171 \mathrm{~Hz})$ at the anomeric position according to the observation of Bock and Pedersen ${ }^{23}$ that $\alpha$-mannosides exhibit anomeric $J_{\mathrm{C}, \mathrm{H}} \sim$ $170 \mathrm{~Hz}$ while $\beta$-mannosides exhibit anomeric $J_{\mathrm{C}, \mathrm{H}} \sim 160 \mathrm{~Hz}$. In every case in our work where a glycosidic bond was formed from mannose, the anomeric configuration of the product(s) was confirmed by this method.

Deacetylation of 18 with methanolic ammonia produced alcohol 19, which was a common intermediate in the synthesis of methyl 1-thioglycoside donors $\mathbf{7 b}$ and $7 \mathbf{c}$ (Schemes 3 and 4).

The synthesis of $\mathbf{7 b}$ was easily realized as shown in Scheme 3. Compound 19 was benzylated to produce $\mathbf{2 0}$ and this was subjected to electrochemical oxidation in aqueous acetonitrile ${ }^{24}$ to remove the $p$-methoxyphenyl (PMP) protecting group providing an anomeric mixture of alcohols 21. This mixture was readily converted into a mixture of anomeric fluorides $\mathbf{2 2}$ by treatment with DAST. ${ }^{25}$ Finally, $\mathrm{SnCl}_{4}$-promoted thiomethy- lation with $\mathrm{Bu}_{3} \mathrm{SnSMe}^{16,26}$ produced the glycosyl donor $\mathbf{7 b}$, ready to couple with acceptor $\mathbf{8}$.

For the synthesis of trisaccharide $\mathbf{7 c}$ (Scheme 4), the common intermediate 19 was glycosylated with methyl 1-thiomannoside 17 to produce trisaccharide 23. Elaboration of $\mathbf{2 3}$ to $7 \mathbf{c}$ proceeded in direct analogy to the synthesis of $\mathbf{7 b}$, producing $7 \mathbf{c}$ in $24 \%$ overall yield from the common intermediate 19.

Synthesis of myo-inositol 11. - The differentially protected inositol 11 (Scheme 5), ready for coupling, was prepared from known, optically pure diol $28^{27}$ by protection of the two free hydroxyl groups as the cyclic carbonate ( $N, N^{\prime}$-carbonyldiimidazole, benzene) followed by removal of the triisopropylsilyl (TIPS) protecting group. Use of acetyl groups to protect the 1- and 2-positions of the inositol ring proved unsuitable because of acetyl group migrations upon fluoride-induced removal of the TIPS group at position 6 . The cyclic carbonate did not suffer migration, presumably because the more rigid structure prevents intramolecular attack of the intermediate C-6 alkoxide on the carbonyl group.

Synthesis of oligosaccharides 1-5. - With the necessary precursors in hand, each final oligosaccharide could be synthesized in seven steps (Scheme 6). The mannose mono-, di-, 


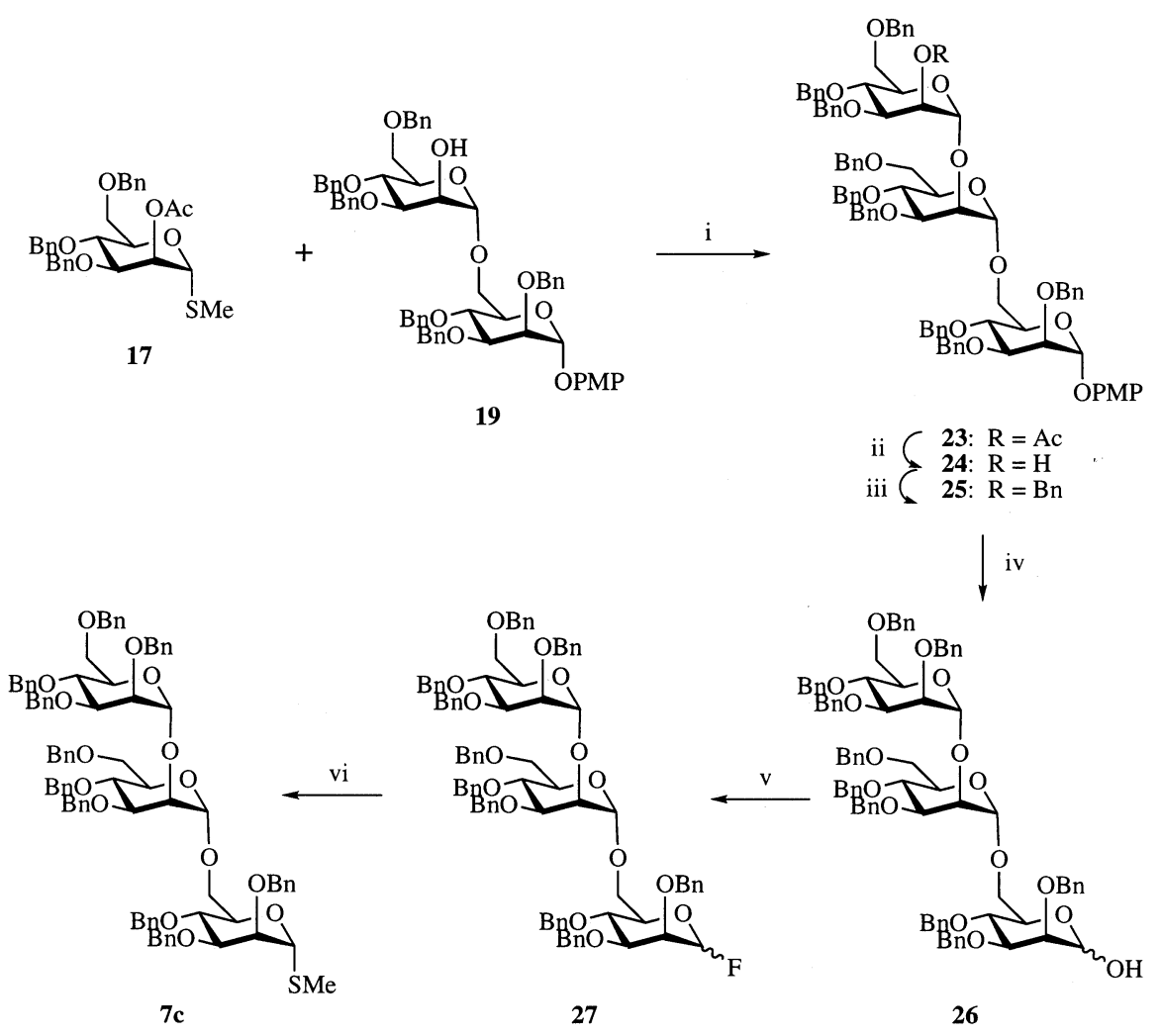

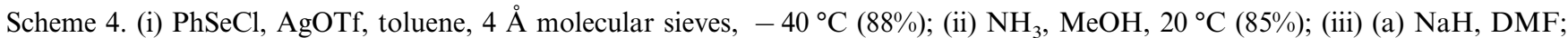
(b) $\mathrm{BnBr}(81 \%)$; (iv) $\mathrm{CH}_{3} \mathrm{CN}-\mathrm{H}_{2} \mathrm{O}$, + $1.55 \mathrm{~V}, 0.1 \mathrm{M} \mathrm{Bu}_{4} \mathrm{NPF}_{6}, 20{ }^{\circ} \mathrm{C}(78 \%)$; (v) DAST, THF (90\%); (vi) $\mathrm{Bu}_{3} \mathrm{SnSMe}, \mathrm{SnCl}{ }_{4}$, $\mathrm{ClCH}_{2} \mathrm{CH}_{2} \mathrm{Cl}(52 \%)$.

and trisaccharides $7 \mathbf{a}-\mathbf{c}$ were coupled with glucosamine derivative $\mathbf{8}$ utilizing Ogawa's method ${ }^{16}$ resulting in $\alpha$-linked disaccharide $\mathbf{9 a}$, trisaccharide $\mathbf{9 b}$, and tetrasaccharide $\mathbf{9 c}$ in 56 , 36 , and $37 \%$ yield, respectively. Fluoride-promoted desilylation of each, followed by conversion into the anomeric fluorides with DAST yielded glycosyl donors suitable for coupling with the inositol. Silver triflate and $\mathrm{Cp}_{2} \mathrm{ZrCl}_{2}$-promoted coupling of $\mathbf{1 1}$ with $\mathbf{1 0 a}-\mathbf{c}$ yielded the corresponding trisaccharide 12a, tetrasaccharide $\mathbf{1 2 b}$, and pentasaccharide 12c. In each case, a mixture of $\alpha$ and $\beta$ anomers was obtained. These were separated chromatographically and each was carried forward independently.

Removal of the cyclic carbonate moiety by alkaline hydrolysis yielded diols ready for installation of the cyclic phosphate moiety. Addition of methylpyridinium dichlorophosphate (31), prepared according to the literature procedure $^{18}$ from methyl phosphorodichloridate and pyridine, to diols 30a $\alpha, 30 \mathbf{a} \beta, 30 \mathbf{b} \alpha$, $30 \mathrm{~b} \beta$, and $30 \mathrm{c} \alpha$ resulted in the formation of the protected oligosaccharide cyclic phosphates. Finally, dissolving-metal reduction ( $\mathrm{Na}(\mathrm{s}), \mathrm{NH}_{3}(\mathrm{l}),-78^{\circ} \mathrm{C}, 15 \mathrm{~min}$ ) followed by careful quenching at $-78^{\circ} \mathrm{C}$ with $\mathrm{NH}_{4} \mathrm{Cl}$ (s), then $\mathrm{MeOH}$, resulted in removal of all benzyl protecting groups and reduction of the azido group to provide compounds 1-5 contaminated with $\mathrm{NaCl}$. Pure products were obtained by desalting with Sephadex G-10 (56-85\% yield).

The biological activity of these compounds is currently being evaluated and will be reported elsewhere.

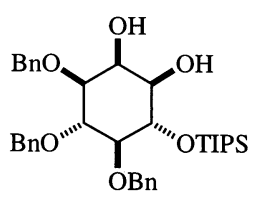

28
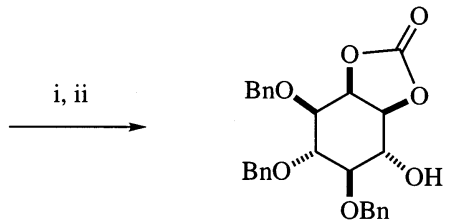

11
Scheme 5. (i) $N, N^{\prime}$-Carbonyldiimidazole, benzene, $20^{\circ} \mathrm{C}$; (ii) TBAF, THF, $0{ }^{\circ} \mathrm{C}, 3 \min (84 \%$, from 28$)$. 

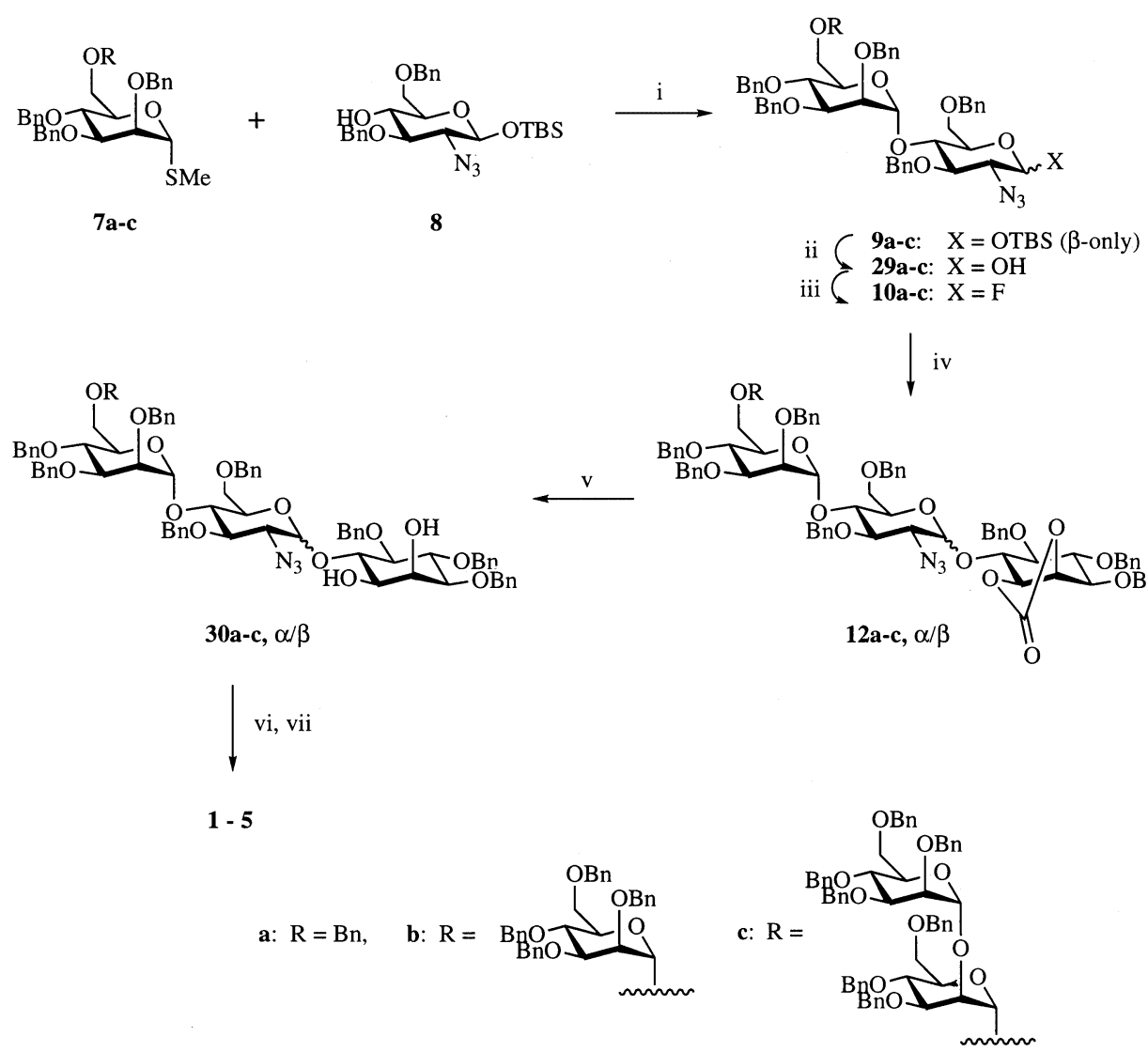

Scheme 6. (i) $\mathrm{PhSeCl}, \mathrm{AgOTf}$, toluene, $4 \AA$ molecular sieves, $-42{ }^{\circ} \mathrm{C}$; (ii) $\mathrm{TBAF}, \mathrm{HOAc}, \mathrm{THF}, 20^{\circ} \mathrm{C}$; (iii) DAST, THF, -42 to $20^{\circ} \mathrm{C}$; (iv) $\mathrm{Cp}_{2} \mathrm{ZrCl}_{2}$, AgOTf, 11, toluene, $4 \AA$ 品 molecular sieves, -42 to $20^{\circ} \mathrm{C}$; (v) $\mathrm{LiOH}, \mathrm{THF}, 12 \mathrm{~h}, 2{ }^{\circ} \mathrm{C}$; (vi) 31 , pyridine; (vii) (a) $\mathrm{Na}, \mathrm{NH}_{3}$ (l), $\mathrm{THF},-78^{\circ} \mathrm{C}, 15 \mathrm{~min}$; (b) $\mathrm{NH}_{4} \mathrm{Cl}$ (s), $-78^{\circ} \mathrm{C}$; (c) $\mathrm{MeOH},-78^{\circ} \mathrm{C}$.

\section{Experimental}

General methods. - All nonaqueous reactions were performed under an Ar atmosphere. Organic extracts were dried with anhyd $\mathrm{MgSO}_{4}$ unless otherwise noted. Solvents were removed in vacuo on a Büchi rotary evaporator. Solvents and reagents obtained from commercial sources were used without further purification with the following exceptions. Tetrahydrofuran (THF) and benzene were distilled from $\mathrm{Na}$ and benzophenone. Acetonitrile, $\mathrm{CH}_{2} \mathrm{Cl}_{2}$, pyridine, and toluene were distilled from $\mathrm{CaH}_{2}$. Benzyl bromide was fractionally distilled. $N, N^{\prime}$ Dimethylformamide (DMF) was dried with $\mathrm{MgSO}_{4}$, filtered, and then distilled. 1,2Dichloroethane was dried with $\mathrm{MgSO}_{4}$, filtered, and distilled from $\mathrm{P}_{2} \mathrm{O}_{5}$. Silver triflate was dried in vacuo $(0.1 \mathrm{mmHg})$ for $24 \mathrm{~h}$. Reactions were monitored by thin-layer chromatography (TLC) on Baker glass-backed silica gel plates $(0.25 \mu \mathrm{m}$ thickness $)$ with a 254 $\mathrm{nm}$ fluorescent indicator. Chromatograms were visualized by one or more of the following techniques: (a) ultraviolet illumination; (b) dipping in an ethanolic solution of $2.5 \% p$-anisaldehyde, $3.5 \% \mathrm{H}_{2} \mathrm{SO}_{4}$ and $1 \% \mathrm{AcOH}$ followed by heating; (c) dipping in an ethanolic solution of Hanes-Isherwood stain (ammonium molybdate- $\mathrm{HCl}$-perchloric acid-acetone) ${ }^{28}$ (d) dipping in a 2-propanol solution of ninhydrin-AcOH-pyridine. ${ }^{28}$ Purifications were performed by flash chromatography on Baker silica gel $(40 \mu \mathrm{m})$, by preparative TLC, or by gel-filtration utilizing Sephadex G-10. Nuclear magnetic resonance (NMR) data were obtained on a Bruker AM-300 FT NMR spectrometer operating at $300 \mathrm{MHz}$ for ${ }^{1} \mathrm{H}$. Tetramethylsilane $\left(\mathrm{Me}_{4} \mathrm{Si}, 0.03 \%\right)$ was used as the internal standard for most ${ }^{1} \mathrm{H}$ and ${ }^{13} \mathrm{C}$ NMR spectra. $\mathrm{CFCl}_{3}$ and $\mathrm{H}_{3} \mathrm{PO}_{4}(85 \%$ in $\mathrm{D}_{2} \mathrm{O}$ ) were used as the external standards for the ${ }^{19} \mathrm{~F}$ and ${ }^{31} \mathrm{P}$ NMR spectra, respectively. High-resolution and low-resolution mass spectrometry data were obtained on a JEOL AX- 
505 or JEOL SX-102 mass spectrometer using FAB or electrospray as the ionization method at the Harvard University Department of Chemistry and Chemical Biology Mass Spectrometry Facility. An EG\&G model 174A polarographic analyzer was used as the potentiostat for the electrochemical oxidations and the $\mathrm{Pt}\left(98 \mathrm{~cm}^{2}\right)$ electrodes were rinsed with concd $\mathrm{HNO}_{3}$ followed by water prior to use.

p - Methoxyphenyl 6 - O - (4,4' - dimethoxytrityl)- $\alpha$-D-mannopyranoside (14). - To a flame-dried flask was added 4,4'-dimethoxytrityl tetrafluoroborate $\left(\mathrm{DMTBF}_{4}, 3.4 \mathrm{~g}\right.$, $8.8 \mathrm{mmol}$ ) and 2,6-di- $t$-butyl-4-methylpyridine (DBMP, $1.8 \mathrm{~g}, 8.8 \mathrm{mmol}$, both were dried by coevaporation with toluene). Acetonitrile (5 $\mathrm{mL}$ ) was added followed by glycoside 13, ${ }^{19,20}$ (1.6 g, 5.9 mmol, previously dried by coevaporation with toluene). The reaction mixture was warmed at reflux. After $12 \mathrm{~h}$ and several additions of more reagent $\left(\mathrm{DMTBF}_{4}\right.$ and $\mathrm{DBMP}$, $3 \times \sim 200 \mathrm{mg}$ ), though the reaction had not gone to completion, the reaction was stopped by addition of $\mathrm{NaHCO}_{3}(1 \mathrm{M}, 10 \mathrm{~mL})$, the mixture extracted with ether $(3 \times 30 \mathrm{~mL})$, and the ethereal extracts were dried, filtered, and concentrated. Purification of the resulting oil via flash-column chromatography utilizing step gradient elution, $\mathrm{CHCl}_{3}$ to remove excess $\mathrm{DMTBF}_{4}$ followed by $9: 1 \mathrm{CHCl}_{3}-\mathrm{MeOH}$, produced $3.2 \mathrm{~g}$ of 14 (92\% yield); $R_{f} 140.37$ (ether); ${ }^{1} \mathrm{H}$ NMR $\left(\mathrm{CDCl}_{3}\right): \delta 2.61(\mathrm{~d}, J 4.0 \mathrm{~Hz}$, $\mathrm{OH}-2), 2.83$ (d, J $5.0 \mathrm{~Hz}, \mathrm{OH}-3), 2.89$ (d, $J \sim 3.0 \mathrm{~Hz}, \mathrm{OH}-4$ ), 3.37 (pseudo-t, $1 \mathrm{H}, J 4.0$ $\mathrm{Hz}), 3.69(\mathrm{~m}, 1 \mathrm{H}), 3.77\left(\mathrm{~s}, 9 \mathrm{H}, \mathrm{OCH}_{3}\right), 3.83$ $(\mathrm{m}, 1 \mathrm{H}), 4.00(\mathrm{~m}, 1 \mathrm{H}, \mathrm{H}-3), 4.11(\mathrm{~m}, 1 \mathrm{H}$, $\mathrm{H}-2$ ), 5.42 (s, $1 \mathrm{H}, \mathrm{H}-1$ ), 6.70-6.85 (pseudo-t, $6 \mathrm{H}, J 7.0 \mathrm{~Hz}), 7.01(\mathrm{~d}, 2 \mathrm{H}, J 7.0 \mathrm{~Hz})$, 7.15-7.35 (m, $7 \mathrm{H}), 7.39$ (d, $2 \mathrm{H}, J 8.0 \mathrm{~Hz})$.

p-Methoxyphenyl 2,3,4-tri-O-benzyl-6-O(4,4 - dimethoxytrityl) - $\alpha$ - D - mannopyranoside (15). - To a solution of $14(3.0 \mathrm{~g}, 5.0 \mathrm{mmol})$ in $\mathrm{DMF}(18.3 \mathrm{~mL})$ at $0{ }^{\circ} \mathrm{C}$ was added $\mathrm{NaH}$ (0.9 $\mathrm{g}, 50 \%$ dispersion in mineral oil). The mixture was allowed to warm to $20^{\circ} \mathrm{C}$ and stirred at that temperature for $25 \mathrm{~min}$. The suspension was again cooled to $0{ }^{\circ} \mathrm{C}$ and $\mathrm{BnBr}$ (3.0 mL, $0.025 \mathrm{~mol}$ ) was added and the mixture was stirred in the dark for $2 \mathrm{~h}$. After the reaction was complete, $\mathrm{NaHCO}_{3}(1 \mathrm{M}, 1 \mathrm{~mL})$ was added and the mixture was extracted with ether $(4 \times 1 \mathrm{~mL})$. The ether layer was washed with $\mathrm{NaHCO}_{3}(1 \mathrm{M}, 4 \times 1 \mathrm{~mL})$, dried, and concentrated. The crude sample was used directly in the next step. A small portion of the crude product was purified for analysis using flash silica gel chromatography with step gradient elution. To prevent hydrolysis, the column was first treated with $1 \%$ triethylamine in hexane. Compound $\mathbf{1 5}$ was purified via flash-column chromatography utilizing step gradient elution: hexane to remove excess $\mathrm{BnBr}$ followed by $2: 1$ hexane-ether to obtain a pure sample of $15 ; R_{f} 150.40(2: 1$ hexanesether); ${ }^{1} \mathrm{H}$ NMR $\left(\mathrm{CDCl}_{3}\right): \delta 3.24(\mathrm{dd}, 1 \mathrm{H}, J$ $5.0 \mathrm{~Hz}), 3.45(\mathrm{~d}, 1 \mathrm{H}, J 10.0 \mathrm{~Hz}), 3.76(\mathrm{~s}, 9 \mathrm{H}$, $\left.\mathrm{OCH}_{3}\right), 3.8-4.1(\mathrm{~m}, 4 \mathrm{H}), 4.3(\mathrm{~d}, 1 \mathrm{H}, J 11.0$ $\mathrm{Hz}), 4.68-4.73$ (m, 4 H), 4.88 (d, $1 \mathrm{H}, J 11.0$ $\mathrm{Hz}$ ), 5.5 (s, $1 \mathrm{H}, \mathrm{H}-1), 6.74-6.82$ (m, $5 \mathrm{H}), 6.9$ $(\mathrm{d}, 2 \mathrm{H}, J 9.0 \mathrm{~Hz}), 7.02(\mathrm{~d}, 2 \mathrm{H}, J 9.0 \mathrm{~Hz})$, 7.17-7.48 (m, aryl H's, $21 \mathrm{H})$.

p-Methoxyphenyl 2,3,4-tri-O-benzyl- $\alpha$-Dmannopyranoside (16). - To a solution of $\mathbf{1 5}$ $(1.0 \mathrm{~g}, 1.0 \mathrm{mmol})$ in $\mathrm{CHCl}_{3}(8 \mathrm{~mL})$ was added a solution of $4 \mathrm{~mL}$ of $1: 1 \mathrm{AcOH}$-water and the mixture was stirred for $2 \mathrm{~h}$ at $80^{\circ} \mathrm{C}$. When the reaction was complete, ether $(25 \mathrm{~mL})$ was added and the solution was neutralized with $\mathrm{NaHCO}_{3}(1 \mathrm{M}, 20 \mathrm{~mL})$. The layers were separated and the aqueous layer was extracted with ether $(3 \times 50 \mathrm{~mL})$. The combined organic extracts were dried, filtered, and concentrated. Compound 16 was purified via flash-column chromatography utilizing step gradient elution: 2:1 hexane-ether was first used to remove nonpolar side-products followed by 1:1 hexane-ether to obtain pure 16 (454 mg, $70 \%$ yield); $R_{f} 160.20$ (1:1 hexanes-ether); ${ }^{1} \mathrm{H}$ NMR $\left(\mathrm{CDCl}_{3}\right): \delta 1.9$ (bs, $\left.1 \mathrm{H}, \mathrm{OH}\right), 3.75$ (s, 3 $\left.\mathrm{H}, \mathrm{OCH}_{3}\right), 3.77(\mathrm{~m}, 2 \mathrm{H}), 3.92(\mathrm{~m}, 1 \mathrm{H})$, 4.05-4.15 (m, 2 H), 4.69-4.77 (m, 5 H, H-2, 4 $\mathrm{H}, \mathrm{CH}_{2} \mathrm{Ph}$ ), 4.82 (d, $1 \mathrm{H}, \mathrm{J} 10.0 \mathrm{~Hz}, \mathrm{CH}_{2} \mathrm{Ph}$ ), $4.93\left(\mathrm{~d}, 1 \mathrm{H}, J 10.0 \mathrm{~Hz}, \mathrm{CH}_{2} \mathrm{Ph}\right), 5.40(\mathrm{~s}, 1 \mathrm{H}$, H-1), 6.78 (d, 2 H, J 9.0 Hz, PMP), 6.88 (d, 2 $\mathrm{H}, J 9.0 \mathrm{~Hz}, \mathrm{PMP}), 7.35-7.45$ (m, $15 \mathrm{H}$, phenyl H's); HRMS: Calcd for $\mathrm{C}_{34} \mathrm{H}_{36} \mathrm{O}_{7} \mathrm{Na}^{+}$: 579.2359; Found: 579.2352.

p-Methoxyphenyl 2-O-acetyl-3,4,6-tri-Obenzyl- $\alpha$-D-mannopyranosyl- $(1 \rightarrow 6)-2,3,4$-triO-benzyl- $\alpha$-D-mannopyranoside (18).-To a flame-dried flask was added $4 \AA$ molecular sieves $(\sim 100 \mathrm{mg})$, phenylselenium chloride 
(0.258 g, $1.349 \mathrm{mmol})$ and toluene $(2 \mathrm{~mL})$. The mixture was cooled to $-30{ }^{\circ} \mathrm{C}\left[\mathrm{CH}_{3} \mathrm{CN}-\right.$ $\mathrm{CO}_{2}(\mathrm{~s})$ ] and silver trifluoromethanesulfonate (462 mg, $1.798 \mathrm{mmol}$ ) was added. Slowly a solution of $16(500 \mathrm{mg}, 0.899 \mathrm{mmol})$ and $17^{16}$ (704 $\mathrm{mg}, 1.349 \mathrm{mmol})$ in toluene $(2 \mathrm{~mL})$ was added to the vigorously stirred mixture. After $\sim 30 \mathrm{~min}, \mathrm{NaHCO}_{3}(1 \mathrm{M}, \sim 5 \mathrm{~mL})$ and bleach $(\sim 5 \mathrm{~mL})$ were added to the reaction mixture. The mixture was stirred until the solution was no longer orange (pale yellow) and extractions were done with ether $(3 \times 5$ $\mathrm{mL})$. The combined organic layers were dried, filtered and concentrated. Compound $\mathbf{1 8}$ was purified via flash-column chromatography utilizing step gradient elution: hexane was first used to remove nonpolar side-products followed by 3:2 hexane-ether to obtain pure $\mathbf{1 8}$ (56-71\% yield); $R_{f} \mathbf{1 7} 0.63, R_{f} \mathbf{1 8} 0.49, R_{f} \mathbf{1 6}$ 0.14 (1:1 hexane-ether); ${ }^{1} \mathrm{H}$ NMR $\left(\mathrm{CDCl}_{3}\right): \delta$ $2.14\left(\mathrm{~s}, 3 \mathrm{H}, \mathrm{CH}_{3} \mathrm{CO}\right), 3.58\left(\mathrm{~s}, 3 \mathrm{H}, \mathrm{CH}_{3} \mathrm{O}\right)$, 3.62 (2pseudo-t, $2 \mathrm{H}, \mathrm{H}-4$ mannose-1, H-4 mannose-2), 3.73 (dd, $1 \mathrm{H}, J 3.7, J 10 \mathrm{~Hz}, \mathrm{H}-3$ mannose-1), 3.82-3.89 (m, $6 \mathrm{H}), 3.96(\mathrm{dd}, 1$ $\mathrm{H}, \mathrm{H}-2$ mannose-1), $4.12(\mathrm{dd}, 1 \mathrm{H}, J 8.9, J 3$ $\mathrm{Hz}, \mathrm{H}-3$ mannose-2), 4.37 (d, $1 \mathrm{H}, J 11 \mathrm{~Hz}$, $\left.\mathrm{CH}_{2} \mathrm{Ph}\right), 4.42\left(\mathrm{~d}, 1 \mathrm{H}, \mathrm{J} 11 \mathrm{~Hz}, \mathrm{CH}_{2} \mathrm{Ph}\right), 4.45$ $\left(\mathrm{d}, 1 \mathrm{H}, J 11 \mathrm{~Hz}, \mathrm{CH}_{2} \mathrm{Ph}\right), 4.51(\mathrm{~d}, 1 \mathrm{H}, J 11$ $\left.\mathrm{Hz}, \mathrm{CH}_{2} \mathrm{Ph}\right), 4.59$ (d, $\left.1 \mathrm{H}, J 11 \mathrm{~Hz}, \mathrm{CH}_{2} \mathrm{Ph}\right)$, $4.66\left(\mathrm{~d}, 1 \mathrm{H}, \mathrm{J} 11 \mathrm{~Hz}, \mathrm{CH}_{2} \mathrm{Ph}\right), 4.68(\mathrm{~s}, 2 \mathrm{H}$, $\left.\mathrm{CH}_{2} \mathrm{Ph}\right), 4.77$ (s, $\left.2 \mathrm{H}, \mathrm{CH}_{2} \mathrm{Ph}\right), 4.86(\mathrm{~d}, 1 \mathrm{H}, \mathrm{J}$ $\left.11 \mathrm{~Hz}, \mathrm{CH}_{2} \mathrm{Ph}\right), 4.90(\mathrm{~d}, 1 \mathrm{H}, J 1.8 \mathrm{~Hz}, \mathrm{H}-2$ mannose-2), 4.93 (d, $\left.1 \mathrm{H}, J 11 \mathrm{~Hz}, \mathrm{CH}_{2} \mathrm{Ph}\right)$, $5.40(\mathrm{~s}, 1 \mathrm{H}, \mathrm{H}-1$ mannose-1), $5.42(\mathrm{~d}, J 1.8$ Hz, H-1 mannose-2), 6.78 (d, $2 \mathrm{H}, J 9 \mathrm{~Hz}$, PMP), 6.98 (d, 2 H, J 9 Hz, PMP), 7.15-7.42 $(\mathrm{m}, 30 \mathrm{H}$, phenyl $\mathrm{H}) ;{ }^{13} \mathrm{C} \mathrm{NMR}\left(\mathrm{CDCl}_{3}\right): \delta 21$ $\left(\mathrm{H}_{3} \mathrm{CCO}\right), 55\left(\mathrm{CH}_{3} \mathrm{O}\right), 66.4\left(\mathrm{CH}_{2}\right), 68.4(\mathrm{CH})$, $68.6(\mathrm{CH}), 71.2(\mathrm{CH}), 71.4\left(\mathrm{CH}_{2}\right), 71.6(\mathrm{CH})$, $72.2\left(\mathrm{CH}_{2}\right), 72.7\left(\mathrm{CH}_{2}\right), 73.2\left(\mathrm{CH}_{2}\right), 74.1(\mathrm{CH})$, $74.5(\mathrm{CH}), 75.0\left(\mathrm{CH}_{2}\right), 77.0\left(\mathrm{t}, \mathrm{CDCl}_{3}\right), 77.8$ $(\mathrm{CH}), 80.0(\mathrm{CH}), 96.6(\mathrm{~d}, J 171 \mathrm{~Hz}, \mathrm{C}-1), 97.8$ (d, J $171 \mathrm{~Hz}, \mathrm{C}-1), 114.5$ (CH, PMP), 117.4 (CH, PMP, 127.6-128.3 (phenyl Cs), 137.9138.6 (phenyl Cs), 150.1 (C-O, PMP), 154.8 (C-O, PMP), $170.1(\mathrm{C}=\mathrm{O})$.

p-Methoxyphenyl 3,4,6-Tri-O-benzyl- $\alpha-\mathrm{D}-$ mannopyranosyl- $(1 \rightarrow 6)-2,3,4$-tri-O-benzyl- $\alpha-$ D-mannopyranoside (19). - Compound 18 (550 $\mathrm{mg}, 0.534 \mathrm{mmol})$ was dissolved in $\mathrm{CH}_{2} \mathrm{Cl}_{2}$ (5 $\mathrm{mL}$ ) and transferred to a pressure vessel.
Methanol (5 mL) was added and the mixture was cooled to $0^{\circ} \mathrm{C}$. Ammonia (g) was bubbled through the solution for $5 \mathrm{~min}$ then the flask was tightly stoppered. The solution was stirred for $12 \mathrm{~h}$ at $20^{\circ} \mathrm{C}$, cooled again to $0^{\circ} \mathrm{C}$ and additional $\mathrm{NH}_{3}$ (g) was added. The reaction was then stirred for $24 \mathrm{~h}$ at $20^{\circ} \mathrm{C}$. The solution was then transferred to a round-bottom flask and concentrated. Purification via silica gel-flash chromatography (1:1 hexane-ether) produced 19 (412 mg, 78\% yield). Crude 19 was not usually purified and was used directly in the production of $20 ; R_{f} \mathbf{1 8} 0.76, R_{f} 190.40$ (1:2 hexane-ether); ${ }^{1} \mathrm{H} \mathrm{NMR}\left(\mathrm{CDCl}_{3}\right): \delta 2.28$ $(\mathrm{s}, 1 \mathrm{H}, \mathrm{OH}), 3.48\left(\mathrm{~s}, 3 \mathrm{H}, \mathrm{CH}_{3} \mathrm{O}\right), 3.50(\mathrm{~s}, 1$ $\mathrm{H}), 3.54-3.57(\mathrm{~m}, 2 \mathrm{H}), 3.63-3.76(\mathrm{~m}, 5 \mathrm{H})$, 3.81-3.82 (m, $2 \mathrm{H}), 3.85-3.86(\mathrm{~m}, 1 \mathrm{H}), 3.95$ $(\mathrm{dd}, 1 \mathrm{H}, J 3, J 11 \mathrm{~Hz}), 4.30(\mathrm{~d}, 2 \mathrm{H}, J 11 \mathrm{~Hz})$, $4.36(\mathrm{~d}, 2 \mathrm{H}, J 11 \mathrm{~Hz}), 4.37(\mathrm{~s}, 1 \mathrm{H}), 4.46(\mathrm{~d}$, $1 \mathrm{H}, J 11 \mathrm{~Hz}), 4.54(\mathrm{~s}, 2 \mathrm{H}), 4.58(\mathrm{~d}, 2 \mathrm{H}), 4.64$ $(\mathrm{d}, 1 \mathrm{H}, J 11 \mathrm{~Hz}), 4.75(\mathrm{~d}, 1 \mathrm{H}, J 11 \mathrm{~Hz}), 4.81$ (s, $1 \mathrm{H}, \mathrm{H}-1), 5.22$ (s, $1 \mathrm{H}, \mathrm{H}-1), 6.53$ (d, $2 \mathrm{H}$, $J 9 \mathrm{~Hz}, \mathrm{PMP}), 6.68$ (d, $2 \mathrm{H}, J 9 \mathrm{~Hz}, \mathrm{PMP})$, 6.91-7.15 (m, 30 H, phenyl H's).

p-Methoxyphenyl 2,3,4,6-tetra-O-benzyl- $\alpha$ D-mannopyranosyl-(1 $\rightarrow 6)$-2,3,4-tri-O-benzyl$\alpha$-D-mannopyranoside (20). - Compound 19 (400 mg, $0.405 \mathrm{mmol}$ ) was dried by coevaporation with toluene, then dissolved in $\mathrm{DMF}$ $(0.4 \mathrm{~mL})$. The solution was cooled to $0^{\circ} \mathrm{C}$ and $\mathrm{NaH}$ (40 mg of a $50 \%$ dispersion in mineral oil, $0.81 \mathrm{mmol})$ was added. The mixture was stirred for $30 \mathrm{~min}$, and then $\mathrm{BnBr}(136 \mathrm{mg}$, $0.096 \mathrm{~mL}, 0.81 \mathrm{mmol}$ ) was added. The mixture was stirred in the dark at $20{ }^{\circ} \mathrm{C}$ and monitored by TLC. When the reaction was complete $(\sim 1 \mathrm{~h}), 5.0 \mathrm{~mL}$ of ether was added and the mixture was washed with $\mathrm{NaHCO}_{3}$ to remove the DMF. The ether layer was dried with $\mathrm{MgSO}_{4}$, filtered, and concentrated. Compound 20 was purified via flash-column chromatography. The crude evaporate was applied to a hexane-equilibrated column as a solution in hexane with a few drops of $\mathrm{CH}_{2} \mathrm{Cl}_{2}$ added to enhance solubility. The column was eluted with a step gradient: hexane was first used to remove excess $\mathrm{BnBr}$ followed by $1.5: 1$ hexane-ether to obtain pure 20 (370 $\mathrm{mg}, 85 \%$ yield); $R_{f} 200.80, R_{f} 190.30$ (1:1 hexanesether); ${ }^{1} \mathrm{H}$ NMR $\left(\mathrm{CDCl}_{3}\right): \delta 3.60(\mathrm{~s}, 3 \mathrm{H}$, $\left.\mathrm{CH}_{3} \mathrm{O}\right), 3.65-4.15$ (m, $12 \mathrm{H}$, mannose), 4.45$4.55(\mathrm{~m}, 4 \mathrm{H}), 4.65-4.82(\mathrm{~m}, 8 \mathrm{H}), 4.88-4.95$ 
$(\mathrm{m}, 2 \mathrm{H}), 5.15(\mathrm{~d}, 1 \mathrm{H}, J 1 \mathrm{~Hz}, \mathrm{H}-1$ mannose2), 5.45 (d, $1 \mathrm{H}, J 1 \mathrm{~Hz}, \mathrm{H}-1$ mannose-1), 6.78 (d, $2 \mathrm{H}, J 9 \mathrm{~Hz}, \mathrm{PMP}), 6.92(\mathrm{~d}, 2 \mathrm{H}, J 9 \mathrm{~Hz}$, PMP), 7.15-7.40 (m, $35 \mathrm{H}$, phenyl H's).

2,3,4,6-Tetra-O - benzyl- $\alpha$ - D - mannopyran osyl- $(1 \rightarrow 6)-2,3,4$-tri-O-benzyl- $\alpha, \beta$-D-mannopyranose (21). - The reaction vessel used was simply a beaker equipped with an $\mathrm{Ag} \mid \mathrm{AgCl}$ reference electrode and two $\mathrm{Pt}\left(98 \mathrm{~cm}^{2}\right)$ electrodes separated by a porous glass plate. Compound 20 (150 mg, $0.139 \mathrm{mmol}$ ) was dissolved in 6:1 MeCN-water $(140 \mathrm{~mL})$ with tetrabutylammonium hexafluorophosphate $\left(\mathrm{Bu}_{4} \mathrm{NPF}_{6}, 0.1 \mathrm{M}, 2.32 \mathrm{~g}\right)$ as the electrolyte. The solution was stirred for $72 \mathrm{~h}$ at $25^{\circ} \mathrm{C}$ as a voltage of $+1.55 \mathrm{~V}$ was applied. When the reaction was complete, the solution was transferred to a round-bottom flask and concentrated; water $(30 \mathrm{~mL})$ was added, and the mixture was extracted with ether $(3 \times 30 \mathrm{~mL})$. The white precipitate, $\mathrm{Bu}_{4} \mathrm{NPF}_{6}$, was collected by filtration for future reuse. The combined ether layers were dried, filtered, and concentrated. Compounds $\mathbf{2 1}$ were purified via flashcolumn chromatography utilizing step gradient elution: 2.5:1 hexane-ether was first used to remove nonpolar side-products followed by 1:2.5 hexane-ether to obtain 21 (100 mg, 74\% yield); $R_{f} 210.20$ (1:1 hexane-ether); ${ }^{1} \mathrm{H}$ NMR $\left(\mathrm{CDCl}_{3}\right): \delta 1.65$ (bs, $\left.1 \mathrm{H}, \mathrm{OH}\right)$, $3.28-3.40,3.55-4.0(2 \mathrm{~m}, 12 \mathrm{H}$, mannose), 4.20-4.75, 4.82-4.90 (2m, $\left.14 \mathrm{H}, \mathrm{CH}_{2} \mathrm{Ph}\right), 5.05$ (s, $1 \mathrm{H}, \mathrm{H}-1$ mannose), 5.15 (s, $1 \mathrm{H}, \mathrm{H}-1$ mannose), 7.10-7.40 (m, $35 \mathrm{H}$, phenyl H's).

2,3,4,6-Tetra-O - benzyl - $\alpha$ - D - mannopyranosyl- $(1 \rightarrow 6)$-2,3,4-tri-O-benzyl- $\alpha, \beta$-D-mannopyranosyl fluoride (22). - Compounds 21 (87 $\mathrm{mg}, 0.089 \mathrm{mmol})$ were azeotropically dried with toluene then dissolved in THF $(0.25 \mathrm{~mL})$ and cooled to $-30^{\circ} \mathrm{C}$. Diethylaminosulfur trifluoride (DAST, $18 \mu \mathrm{L}, 0.135 \mathrm{mmol}$ ) was added and the reaction mixture was slowly brought to $20^{\circ} \mathrm{C}$ over $30 \mathrm{~min}$. When the reaction was complete, the mixture was cooled to $-30{ }^{\circ} \mathrm{C}$ and $\mathrm{MeOH}$ was added to quench any unreacted DAST. After warming the mixture to $20^{\circ} \mathrm{C}, \mathrm{NaHCO}_{3}(1 \mathrm{M}, \sim 1 \mathrm{~mL})$ was added and the mixture was extracted with ether $(3 \times 5 \mathrm{~mL})$. The combined ether layers were dried, filtered, and concentrated. Flash silica gel-column chromatography (3:1 hex- ane-ether) was used to obtain 22 (75 mg, 86\% yield), $R_{f} \quad 220.63$ (1:1 hexanes-ether); ${ }^{1} \mathrm{H}$ NMR $\left(\mathrm{CDCl}_{3}\right): \delta 3.60-3.78,3.80-4.00(2 \mathrm{~m}$, $12 \mathrm{H}$, mannose), 4.45-4.75, 4.80-5.20 (2m, 14 $\left.\mathrm{H}, \mathrm{CH}_{2} \mathrm{Ph}\right), 5.52$ (d, $\left.1 \mathrm{H}, J 50 \mathrm{~Hz}, \mathrm{H}-1\right)$, 7.10-7.40 (m, 35 H, phenyl H's).

Methyl 2,3,4,6-tetra-O-benzyl- $\alpha$-D-mannopyranosyl- $(1 \rightarrow 6)-2,3,4-$ tri-O-benzyl-1-thio- $\alpha$ D-mannopyranoside (7b). - To pre-dried 22 (140 mg, $0.144 \mathrm{mmol}$ ) cooled to $0{ }^{\circ} \mathrm{C}$ was added $\mathrm{Bu}_{3} \mathrm{SnSMe}$ in $\mathrm{CH}_{2} \mathrm{ClCH}_{2} \mathrm{Cl}(97 \mathrm{mg}$, $0.288 \mathrm{mmol}, 1.3 \mathrm{~mL}$ of a $74 \mathrm{mg} / \mathrm{mL}$ stock solution). After stirring for $5 \mathrm{~min}, \mathrm{SnCl}_{4}$ in $\mathrm{CH}_{2} \mathrm{ClCH}_{2} \mathrm{Cl}(75 \mathrm{mg}, 0.29 \mathrm{mmol}, 1.3 \mathrm{~mL}$ of a $57 \mathrm{mg} / \mathrm{mL}$ stock solution) was added. The yellowish solution was stirred at $0{ }^{\circ} \mathrm{C}$ for 15 min. When the reaction was complete, $\mathrm{NaF}$ (1 $\mathrm{M}, 15 \mathrm{~mL}$ ) and EtOAc were added and the reaction was stirred for $1 \mathrm{~h}$. The resulting white precipitate was removed by filtration through Celite. The organic layer was separated, washed with $\mathrm{NaHCO}_{3}(1 \mathrm{M}, 3 \times 15$ $\mathrm{mL}$ ), dried, filtered and concentrated. To obtain pure product, two flash chromatography separations were required. After the first separation (8:1 toluene-EtOAc, $R_{f} \alpha, \beta$ RSMe $0.80)$, the mixture of anomers was collected and applied to a second column (1:1 hexanesether) to produce pure $7 \mathbf{b}(122 \mathrm{mg}, 85 \%$ yield $)$. For 7b: $R_{f} 0.56$ (1:1 hexanes-ether); ${ }^{1} \mathrm{H}$ NMR $\left(\mathrm{CDCl}_{3}\right): \delta 2.05\left(\mathrm{~s}, 3 \mathrm{H}, \mathrm{CH}_{3} \mathrm{~S}\right), 3.62-4.05(\mathrm{~m}$, $12 \mathrm{H}$, mannose), 4.55-4.70 (m, $\left.13 \mathrm{H}, \mathrm{CH}_{2} \mathrm{Ph}\right)$, $4.88\left(\mathrm{~d}, \mathrm{~J} 12 \mathrm{~Hz}, \mathrm{CH} \mathrm{H}_{2} \mathrm{Ph}\right.$ ), 5.10 (s, $\left.1 \mathrm{H}, \mathrm{H}-1\right)$, 5.20 (s, $1 \mathrm{H}, \mathrm{H}-1), 7.10-7.38$ (m, $35 \mathrm{H}$, phenyl H's).

For the $\beta$ anomer: $R_{f} 0.33 ;{ }^{1} \mathrm{H}$ NMR $\left(\mathrm{CDCl}_{3}\right): \delta 2.18\left(\mathrm{~s}, 3 \mathrm{H}, \mathrm{CH}_{3} \mathrm{~S}\right), 3.35,3.58-$ 4.05 (2m, mannose), 4.39-4.98 (m, $\mathrm{CH}_{2} \mathrm{Ph}$ and mannose), $5.15(\mathrm{~d}, 1 \mathrm{H}, J 1.5 \mathrm{~Hz}, \mathrm{H}-1$ mannose-2), 7.15-7.48 (m, phenyl H's).

p-Methoxyphenyl 2-O-acetyl-3,4,6-tri-Obenzyl- $\alpha$-D-mannopyranosyl- $(1 \rightarrow 2)-3,4,6$-triO-benzyl- $\alpha$-D-mannopyranosyl- $(1 \rightarrow 6)-2,3,4$ tri-O-benzyl- $\alpha$-D-mannopyranosidee $\quad(\mathbf{2 3})$.Compound $\mathbf{2 3}$ was synthesized in a manner analogous to the synthesis of compound $\mathbf{1 8}$ except that 19 was used as the glycosyl acceptor in place of 16. The trisaccharide product 23 was purified via flash chromatography utilizing step gradient elution: hexane was first used to remove nonpolar 
side-products followed by 3:1 hexane-ether to obtain pure 23 (88\% yield); $R_{f} 230.47$ (1:1 hexanes-ether); ${ }^{1} \mathrm{H}$ NMR $\left(\mathrm{CDCl}_{3}\right): \delta 2.12(\mathrm{~s}$, $\left.3 \mathrm{H}, \mathrm{CH}_{3} \mathrm{CO}\right), 3.58\left(\mathrm{~s}, 3 \mathrm{H}, \mathrm{CH}_{3} \mathrm{O}\right), 3.50-4.20$ (m, $17 \mathrm{H}$, mannose), 4.10 (dd, $1 \mathrm{H}, J 3, J 11$ $\mathrm{Hz}), 4.34$ (d, $\left.1 \mathrm{H}, J 12 \mathrm{~Hz}, \mathrm{CH}_{2} \mathrm{Ph}\right), 4.43$ (d, 1 $\left.\mathrm{H}, J 12 \mathrm{~Hz}, \mathrm{CH}_{2} \mathrm{Ph}\right), 4.48(\mathrm{~d}, 1 \mathrm{H}, J 12 \mathrm{~Hz}$, $\left.\mathrm{CH}_{2} \mathrm{Ph}\right), 4.52\left(\mathrm{~s}, 2 \mathrm{H}, \mathrm{CH}_{2} \mathrm{Ph}\right), 4.56(\mathrm{~d}, 1 \mathrm{H}, \mathrm{J}$ $\left.12 \mathrm{~Hz}, \mathrm{CH}_{2} \mathrm{Ph}\right), 4.62$ (d, $1 \mathrm{H}, J 12 \mathrm{~Hz}$, $\left.\mathrm{CH}_{2} \mathrm{Ph}\right), 4.63\left(\mathrm{~d}, 1 \mathrm{H}, J 12 \mathrm{~Hz}, \mathrm{CH}_{2} \mathrm{Ph}\right), 4.67$ (s, $\left.2 \mathrm{H}, \mathrm{CH}_{2} \mathrm{Ph}\right), 4.75$ (s, $\left.2 \mathrm{H}, \mathrm{CH}_{2} \mathrm{Ph}\right), 4.82$ (d, $\left.1 \mathrm{H}, J 12 \mathrm{~Hz}, \mathrm{CH}_{2} \mathrm{Ph}\right), 4.86(\mathrm{~d}, J 12 \mathrm{~Hz}$, $\left.\mathrm{CH}_{2} \mathrm{Ph}\right), 4.91\left(\mathrm{~d}, 1 \mathrm{H}, \mathrm{J} 12 \mathrm{~Hz}, \mathrm{CH}_{2} \mathrm{Ph}\right), 4.93$ (pseudo-t, $1 \mathrm{H}, J 1 \mathrm{~Hz}, \mathrm{H}-1$ mannose-2), 5.03 (d, $1 \mathrm{H}, J 1 \mathrm{~Hz}, \mathrm{H}-1$ mannose-3), 5.41 (d, $1 \mathrm{H}$, $J 1 \mathrm{~Hz}, \mathrm{H}-1$ mannose-1), 5.52 (pseudo-t, $1 \mathrm{H}$, $J 1 \mathrm{~Hz}, \mathrm{H}-2$ mannose-3), 6.70 (d, $2 \mathrm{H}, J 9 \mathrm{~Hz}$, PMP), 6.93 (d, 2 H, J 9 Hz, PMP), 7.10-7.42 (m, $45 \mathrm{H}$, phenyl H's); ${ }^{13} \mathrm{C} \mathrm{NMR}\left(\mathrm{CDCl}_{3}\right): \delta$ $21\left(\mathrm{CH}_{3} \mathrm{CO}\right), 53.45,55.44\left(\mathrm{CH}_{3} \mathrm{O}\right), 66.49$, 68.83, 71.88, 72.30, 72.34, 73.30, 74.62, 75.02, $77.04\left(\mathrm{t}, \mathrm{CDCl}_{3}\right) 78.18,79.32,80.15,96.94(\mathrm{~d}$, $J 170 \mathrm{~Hz}, \mathrm{C}-\mathrm{H}, \mathrm{C}-1), 98.81$ (d, J $171 \mathrm{~Hz}$, C-H, C-1), 99.57 (d, J 173 Hz, C-H, C-1), 114.64 (CH, PMP), 117.52 (CH, PMP), 127.55-133.58 (phenyl Cs), 137.9-140.0 (aromatic), 151 (C-O, PMP), 156 (C-O, PMP), $171(\mathrm{C}=\mathrm{O})$; HRMS: Calcd for $\mathrm{C}_{90} \mathrm{H}_{94} \mathrm{O}_{18}+$ Na: 1485.6337; Found: 1485.4656.

p-Methoxyphenyl 3,4,6-tri-O - benzyl- $\alpha$ - Dmannopyranosyl- $(1 \rightarrow 2)-3,4,6$ - tri-O-benzyl- $\alpha-$ D-mannopyranosyl-(1 $\rightarrow 6)$-2,3,4-tri-O-benzyl$\alpha$-D-mannopyranoside (24). - Compound 24 was synthesized in a manner analogous to compound 19 except that compound 23 was deacetylated instead of compound 18. After work-up, silica gel-flash chromatography (1:1 hexane-ether) was used to obtain pure product 24 (554 $\mathrm{mg}, 85 \%$ yield); $R_{f} \mathbf{2 4} 0.20$ (1:1 hexanes-ether); ${ }^{1} \mathrm{H}$ NMR $\left(\mathrm{CDCl}_{3}\right): \delta 2.45$ (s, $1 \mathrm{H}, \mathrm{OH}), 3.58\left(\mathrm{~s}, 3 \mathrm{H}, \mathrm{CH}_{3} \mathrm{O}\right), 3.52-3.98$ (m, $24 \mathrm{H}$, mannose), 4.05 ( $\mathrm{m}, 1 \mathrm{H}, \mathrm{H}-2$ mannose-3), 4.09 (d, $1 \mathrm{H}, J 2 \mathrm{~Hz}), 4.11$ (d, $1 \mathrm{H}, J$ $1 \mathrm{~Hz}), 4.34$ (d, $\left.1 \mathrm{H}, J 12 \mathrm{~Hz}, \mathrm{CH}_{2} \mathrm{Ph}\right), 4.45$ (d, $\left.1 \mathrm{H}, J 12 \mathrm{~Hz}, \mathrm{CH}_{2} \mathrm{Ph}\right), 4.47$ (d, $1 \mathrm{H}, J 12 \mathrm{~Hz}$, $\left.\mathrm{CH}_{2} \mathrm{Ph}\right), 4.50\left(\mathrm{~d}, 1 \mathrm{H}, \mathrm{J} 12 \mathrm{~Hz}, \mathrm{CH}_{2} \mathrm{Ph}\right), 4.52$ (d, $\left.1 \mathrm{H}, \mathrm{J} 12 \mathrm{~Hz}, \mathrm{CH}_{2} \mathrm{Ph}\right), 4.54$ (d, $1 \mathrm{H}, J 12$ $\left.\mathrm{Hz}, \mathrm{CH}_{2} \mathrm{Ph}\right), 4.56$ (d, $1 \mathrm{H}, \mathrm{J} 12 \mathrm{~Hz}, \mathrm{CH}_{2} \mathrm{Ph}$ ), $4.58\left(\mathrm{~d}, 1 \mathrm{H}, J 12 \mathrm{~Hz}, \mathrm{CH}_{2} \mathrm{Ph}\right), 4.63(\mathrm{~d}, 1 \mathrm{H}$, $\left.J 12 \mathrm{~Hz}, \mathrm{CH}_{2} \mathrm{Ph}\right), 4.68(\mathrm{~d}, 1 \mathrm{H}, J 12 \mathrm{~Hz}$, $\left.\mathrm{CH}_{2} \mathrm{Ph}\right), 4.70$ (s, $\left.2 \mathrm{H}, \mathrm{CH}_{2} \mathrm{Ph}\right), 4.77$ (s, $2 \mathrm{H}$,
$\left.\mathrm{CH}_{2} \mathrm{Ph}\right), 4.82\left(\mathrm{~d}, 1 \mathrm{H}, \mathrm{J} 12 \mathrm{~Hz}, \mathrm{CH}_{2} \mathrm{Ph}\right), 4.88$ (d, J $\left.12 \mathrm{~Hz}, \mathrm{CH}_{2} \mathrm{Ph}\right), 4.92$ (d, $1 \mathrm{H}, J 12 \mathrm{~Hz}$, $\mathrm{CH}_{2} \mathrm{Ph}$ ), 5.1 (pseudo-s, $1 \mathrm{H}, \mathrm{H}-1$ mannose), 5.2 (pseudo-s, $1 \mathrm{H}, \mathrm{H}-1$ mannose), 5.5 (pseudo-s, $1 \mathrm{H}, \mathrm{H}-1$ mannose), 6.78 (d, $2 \mathrm{H}, J$ $9 \mathrm{~Hz}, \mathrm{PMP}$ ), 7.00 (d, $2 \mathrm{H}, J 9 \mathrm{~Hz}, \mathrm{PMP})$, 7.15-7.45 (m, $45 \mathrm{H}$, phenyl H's).

p-Methoxyphenyl 2,3,4,6-tetra-O-benzyl- $\alpha$ D-mannopyranosyl-(1 $\rightarrow$ 2)-3,4,6-tri-O-benzyl$\alpha$-D-mannopyranosyl-(1 $\rightarrow 6)-2,3,4$-tri-O-benzyl- $\alpha$-D-mannopyranoside (25).-Compound 25 was synthesized in a manner analogous to synthesis of compound $\mathbf{2 0}$ except that alcohol 24 was benzylated instead of alcohol 19. After work-up, product $\mathbf{2 5}$ was purified via flashcolumn chromatography utilizing step gradient elution: hexane was used first to remove nonpolar side-products followed by $2: 1$ hexane-ether to obtain pure $\mathbf{2 5}$ (81\% yield); $R_{f} \mathbf{2 5}$ 0.49 (1:1 hexanes-ether); ${ }^{1} \mathrm{H}$ NMR $\left(\mathrm{CDCl}_{3}\right): \delta$ $3.55\left(\mathrm{~s}, 3 \mathrm{H}, \mathrm{CH}_{3} \mathrm{O}\right), 3.60-4.12(\mathrm{~m}, 18 \mathrm{H}$, mannose), 4.42-4.90 (m, $\left.20 \mathrm{H}, \mathrm{CH}_{2} \mathrm{Ph}\right), 4.93$ (m, $1 \mathrm{H}, \mathrm{H}-1), 5.13$ (d, $1 \mathrm{H}, J 1 \mathrm{~Hz}, \mathrm{H}-1), 5.42$ (d, $1 \mathrm{H}, J 2 \mathrm{~Hz}, \mathrm{H}-1), 6.75$ (d, $2 \mathrm{H}, J 9 \mathrm{~Hz}$, PMP), 6.95 (d, 2 H, J 9 Hz, PMP), 7.15-7.45 (m, $50 \mathrm{H}$, aromatic).

2,3,4,6-Tetra - O - benzyl- $\alpha$ - D - mannopyran osyl- $(1 \rightarrow 2)$ - 3,4,6-tri-O - benzyl- $\alpha$ - D - mannopyranosyl- $(1 \rightarrow 6)-2,3,4-$ tri-O - benzyl $-\alpha, \beta$ - Dmannopyranose (26). - Compound 26 were synthesized by a procedure analogous to that utilized for the synthesis of compounds $\mathbf{2 1}$ except that trisaccharide $\mathbf{2 5}$ was oxidized instead of disaccharide 20. After work-up, product $\mathbf{2 6}$ was purified via flash-column chromatography utilizing step gradient elution: 2:1 hexane-ether was used first to remove nonpolar side-products followed by 1:2 hexaneether to obtain 26 (78\% yield); $R_{f} 260.18$ (1:1 hexanes-ether); ${ }^{1} \mathrm{H}$ NMR $\left(\mathrm{CDCl}_{3}\right): \delta 3.60-$ $4.00(\mathrm{~m}, 17 \mathrm{H}$, mannose $), 4.18(\mathrm{~m}, 1 \mathrm{H})$, 4.45-4.90 (m, $\left.20 \mathrm{H}, \mathrm{CH}_{2} \mathrm{Ph}\right), 4.92(\mathrm{~m}, 1 \mathrm{H}$, H-1), 4.96 (d, $1 \mathrm{H}, J 2 \mathrm{~Hz}, \mathrm{H}-1), 5.16$ (d, $1 \mathrm{H}$, $J 1 \mathrm{~Hz}, \mathrm{H}-1), 7.15-7.40$ (m, $50 \mathrm{H}$, aromatic).

2,3,4,6-Tetra-O - benzyl - $\alpha$ - D - mannopyranosyl- $(1 \rightarrow 2)$ - 3,4,6-tri-O - benzyl - $\alpha$ - D - manno pyranosyl- $(1 \rightarrow 6)-2,3,4$ - tri - O - benzyl - $\alpha, \beta$ - Dmannopyranosyl fluoride (27)._-Compound 27 were synthesized in a manner analogous to the synthesis of compound 22 except that trisaccharide $\mathbf{2 6}$ were fluorinated instead of di- 
saccharide 21. After work-up, flash silica gelcolumn chromatography (2:1 hexanes-ether) was used to obtain pure 27 (as an anomeric mixture), 90\% yield; $R_{f} 270.62$ (1:1 hexanesether); ${ }^{1} \mathrm{H} \mathrm{NMR}\left(\mathrm{CDCl}_{3}\right): \delta 3.55-4.03(\mathrm{~m}, 17$ $\mathrm{H}$, mannose), 4.15 (pseudo-t, $1 \mathrm{H}, J 2 \mathrm{~Hz}$ ), 4.40-4.89 (m, $\left.20 \mathrm{H}, \mathrm{CH}_{2} \mathrm{Ph}\right), 4.96$ (d, $1 \mathrm{H}, J 1$ $\mathrm{Hz}, \mathrm{H}-1), 5.15$ (d, $1 \mathrm{H}, J 1 \mathrm{~Hz}, \mathrm{H}-1), 5.56$ (d, $1 \mathrm{H}, J 51 \mathrm{~Hz}, \mathrm{H}-1$ mannose-1), 7.10-7.36 (m, $50 \mathrm{H}$, phenyl H's); ${ }^{19} \mathrm{~F}$ NMR $\left(\mathrm{CDCl}_{3}\right) \delta-$ $138.5\left(\mathrm{~d}, J_{\mathrm{FH}} 51 \mathrm{~Hz}\right)$.

Methyl 2,3,4,6-tetra-O-benzyl- $\alpha$-D-mannopyranosyl - $(1 \rightarrow 2)-3,4,6$ - tri - O - benzyl - $\alpha$ - Dmannopyranosyl- $(1 \rightarrow 6)-2,3,4$-tri-O-benzyl-1thio - $\alpha, \beta$ - D - mannopyranoside (7c). - Compound 7c was synthesized in a manner analogous to the synthesis of compound $\mathbf{7 b}$ except that trisaccharides 27 were thiomethylated instead of disaccharides 22. To obtain the pure $\alpha$ anomer, two flash chromatography column separations were required. After the first column (8:1 toluene-EtOAc, $R_{f} \quad \alpha, \beta$ RSMe $\sim 0.80$ ), the mixture of anomers were collected and applied to a second column (3:2 hexanes-ether) providing $80 \mathrm{mg}$ (52\% yield) of 7c ( $\alpha$ anomer) and $7 \mathrm{mg}$ (5\% yield) of the corresponding $\beta$ anomer; $R_{f}$ 7c 0.50 (1:1 hexanes-ether); ${ }^{1} \mathrm{H}$ NMR 7c $\left(\mathrm{CDCl}_{3}\right): \delta 2.05$ (s, 3 $\left.\mathrm{H}, \mathrm{CH}_{3} \mathrm{~S}\right), 3.55-3.95,4.05-4.10$ (2m, mannose), 4.15 (pseudo-t, $1 \mathrm{H}, J 2 \mathrm{~Hz}$ ), 4.45-4.95 $\left(\mathrm{m}, 20 \mathrm{H}, \mathrm{CH}_{2} \mathrm{Ph}\right), 4.95(\mathrm{~d}, 1 \mathrm{H}, J 1.5 \mathrm{~Hz}$, $\mathrm{H}-1), 5.18$ (d, $1 \mathrm{H}, J 1.5 \mathrm{~Hz}, \mathrm{H}-1), 7.15-7.42$ (m, $50 \mathrm{H}$, phenyl H's); $R_{f}$ ( $\beta$ anomer) 0.25 (1:1 hexanes-ether); ${ }^{1} \mathrm{H}$ NMR ( $\beta$ anomer, $\left.\mathrm{CDCl}_{3}\right)$ : $\delta$ 3.40, 3.55-4.05 (2m, mannose), 4.10 (pseudo-t, $1 \mathrm{H}, J 1 \mathrm{~Hz}), 4.40-4.95\left(\mathrm{~m}, \mathrm{CH}_{2} \mathrm{Ph}\right.$ and mannose), 5.20 (d, $1 \mathrm{H}, J 1 \mathrm{~Hz}, \mathrm{H}-1), 5.30$ (d, $1 \mathrm{H}, J 1 \mathrm{~Hz}, \mathrm{H}-1), 5.30\left(\mathrm{CH}_{2} \mathrm{Cl}_{2}\right), 7.10-$ 7.40 (m, $50 \mathrm{H}$, phenyl H's).

3,4,5 - Tri - O - benzyl - 1D - myo - inositol 1,2cyclic carbonate (11). - To $335 \mathrm{mg}$ of diol $\mathbf{2 8}^{27}$ $(0.55 \mathrm{mmol})$ in $27.5 \mathrm{~mL}$ of benzene was added $275 \mathrm{mg}$ of $1,1^{\prime}$-carbonyldiimidazole (1.7 $\mathrm{mmol})$. The reaction was stirred at $20^{\circ} \mathrm{C}$ for $17 \mathrm{~h}$ and quenched by the addition of water $(20 \mathrm{~mL})$. The layers were separated and the aqueous layer was extracted with $\mathrm{CH}_{2} \mathrm{Cl}_{2}(4 \times$ $20 \mathrm{~mL})$. The combined organic extracts were washed with satd $\mathrm{NaCl}(20 \mathrm{~mL})$, dried, concentrated by co-evaporation with toluene, and dissolved in $24 \mathrm{~mL}$ of dry THF. The reaction was cooled to $0{ }^{\circ} \mathrm{C}$ and $5.5 \mathrm{~mL}$ of $1 \mathrm{M} t$-butylammonium fluoride (TBAF) in THF was added. The reaction was stirred at $0{ }^{\circ} \mathrm{C}$ for 3 min and quenched by addition of $12 \mathrm{~mL}$ of water at $0{ }^{\circ} \mathrm{C}$. The mixture was extracted with $\mathrm{CH}_{2} \mathrm{Cl}_{2}(4 \times 12 \mathrm{~mL})$. The combined organic extracts were washed with water $(2 \times 10 \mathrm{~mL})$ and then satd $\mathrm{NaCl}(10 \mathrm{~mL})$, dried, and concentrated. Purification via flash silica-column chromatography yielded $262 \mathrm{mg}$ of 11 (84\%). $[\alpha]_{\mathrm{D}}+22.7^{\circ}\left(c \quad 0.0075, \mathrm{CH}_{2} \mathrm{Cl}_{2}\right) ; R_{f} \mathbf{1 1} 0.29$ (7:3 hexanes-EtOAc); ${ }^{1} \mathrm{H}$ NMR $\left(\mathrm{CDCl}_{3}\right): \delta$ 2.7 (bs, $1 \mathrm{H}, \mathrm{OH}), 3.38$ (dd, $1 \mathrm{H}, J 4.5,10.7$ $\mathrm{Hz}), 3.79$ (dd, $1 \mathrm{H}, J$ 2.5, $4.5 \mathrm{~Hz}), 3.90$ (pseudo-t, $1 \mathrm{H}, J 2.8 \mathrm{~Hz}$ ), 4.31 (d, $1 \mathrm{H}, J 11.5$ $\mathrm{Hz}, H \mathrm{CHPh}), 4.38$ (d, $1 \mathrm{H}, J 11.5 \mathrm{~Hz}$, $H \mathrm{CHPh}), 4.45$ (dd, $1 \mathrm{H}, J$ 7.0, $10.7 \mathrm{~Hz}), 4.56$ (d, $1 \mathrm{H}, J 11.4 \mathrm{~Hz}, H \mathrm{CHPh}), 4.58$ (d, $1 \mathrm{H}, J$ $11.9 \mathrm{~Hz}, H \mathrm{CHPh}), 4.62-2.67(\mathrm{~m}, 2 \mathrm{H}), 4.71$ (d, $1 \mathrm{H}, J 11.9 \mathrm{~Hz}, H \mathrm{CHPh}), 4.86$ (dd, $1 \mathrm{H}, J$ $3.3,8.7 \mathrm{~Hz}), 7-7.5(\mathrm{~m}, 15 \mathrm{H}$, phenyl H's $) ;{ }^{13} \mathrm{C}$ NMR $\left(\mathrm{CDCl}_{3}\right): \delta 70.57(\mathrm{C}-\mathrm{O}), 72.02(\mathrm{C}-\mathrm{O})$, $73.34(\mathrm{C}-\mathrm{O}), 73.43(\mathrm{C}-\mathrm{O}), 73.72(\mathrm{C}-\mathrm{O}), 74.34$ $(\mathrm{C}-\mathrm{O}), \quad 79.26 \quad(\mathrm{C}-\mathrm{O}), \quad 80.07 \quad(\mathrm{C}-\mathrm{O}), 80.92$ (C-O), 127.89 (phenyl C), 128.15 (phenyl C), 128.52 (phenyl C), 136.76 (phenyl C), 137.46 (phenyl C), $154.46 \quad(\mathrm{C}=\mathrm{O})$. HRMS-FAB ${ }^{+}$: Calcd for $\mathrm{C}_{28} \mathrm{H}_{28} \mathrm{O}_{7}+\mathrm{Na}$ 499.1733; Found 499.1744.

$\mathrm{t}$-Butyldimethylsilyl 2,3,4,6-tetra-O-benzyl$\alpha$ - D - mannopyranosyl- $(1 \rightarrow 4)$ - 2 - azido - 2 - de oxy - 3,6 - di - O - benzyl - $\beta$ - D - glucopyranoside (9a). - To a flame-dried flask containing a stir bar was added $\mathrm{PhSeCl}$ (60 mg, $0.313 \mathrm{mmol}), 4$ $\AA$ molecular sieves, and $2.4 \mathrm{~mL}$ of toluene. The reaction mixture was cooled to $0{ }^{\circ} \mathrm{C}$ and AgOTf (80 mg, $0.311 \mathrm{mmol})$ was added. After stirring at $0{ }^{\circ} \mathrm{C}$ for $10 \mathrm{~min}$, the mixture was cooled to $-42{ }^{\circ} \mathrm{C}$ and a solution containing 7a (120 mg, $0.206 \mathrm{mmol})$ and $\mathbf{8}^{15}$ (86 mg, 0.171 $\mathrm{mmol})$, pre-dried by coevaporation with toluene, in $7.5 \mathrm{~mL}$ of toluene was added. The reaction was stirred at $-42{ }^{\circ} \mathrm{C}$ for $30 \mathrm{~min}$, then quenched by addition of $1 \mathrm{M} \mathrm{NaHCO}$ $(5 \mathrm{~mL})$ followed by filtration through Celite. The layers were separated, the filtrate was diluted with $\mathrm{CHCl}_{3}(5 \mathrm{~mL})$ and the organic layer was washed with water $(3 \times 5 \mathrm{~mL})$, dried, and concentrated. Purification via silica gel chromatography with $4: 1$ hexane-ether provided $99 \mathrm{mg}$ of $9 \mathbf{a}$ as an oil (56\% yield); $R_{f}$ 9a $0.26 \quad(4: 1$ hexane-ether $) ;{ }^{1} \mathrm{H} \quad \mathrm{NMR}$ 
$\left(\mathrm{CDCl}_{3}\right): \delta 0.19\left(\mathrm{~s}, 3 \mathrm{H}, \mathrm{CH}_{3}\right), 0.20(\mathrm{~s}, 3 \mathrm{H}$, $\left.\mathrm{CH}_{3}\right), 0.94$ (s, $9 \mathrm{H}, t$-butyl), $3.25(\mathrm{dd}, 1 \mathrm{H}, J$ 8.6, $9.9 \mathrm{~Hz}, \mathrm{H}-3$ glucosamine), 3.36-3.42 (m, $2 \mathrm{H}, \mathrm{H}-5$ and $\mathrm{H}-2$ of glucosamine), 3.58 (dd, 1 $\mathrm{H}, J 1.5,9.1 \mathrm{~Hz}, \mathrm{H}-3$ mannose), 3.64-3.83 (m, $7 \mathrm{H}$ ), 3.98 (pseudo-t, $1 \mathrm{H}, J 9.3 \mathrm{~Hz}, \mathrm{H}-4$ mannose), 4.25 (d, $1 \mathrm{H}, J 12.2 \mathrm{~Hz}, H \mathrm{CHPh})$, 4.35 (d, $1 \mathrm{H}, J 12.2 \mathrm{~Hz}, H \mathrm{CHPh}), 4.43-4.63$ $(\mathrm{m}, 9 \mathrm{H}), 4.82(\mathrm{~d}, 1 \mathrm{H}, J 10.8 \mathrm{~Hz}, H \mathrm{CHPh})$, $4.94(\mathrm{~d}, 1 \mathrm{H}, J 11.4 \mathrm{~Hz}, H \mathrm{CHPh}), 5.30$ (d, 1 $\mathrm{H}, J 2.0 \mathrm{~Hz}, \mathrm{H}-1$ mannose), 7.14-7.40 (m, phenyl H's); ${ }^{13} \mathrm{C} \mathrm{NMR}\left(\mathrm{CDCl}_{3}\right): \delta 100.15(\mathrm{~d}, J$ $169 \mathrm{~Hz}, \mathrm{C}-1$ mannose), 97.15 (d, J $160 \mathrm{~Hz}$, C-1 glucosamine).

$\mathrm{t}$-Butyldimethyl 2,3,4,6-tetra-O-benzyl- $\alpha$-Dmannopyranosyl- $(1 \rightarrow 6)-2,3,4$-tri-O-benzyl- $\alpha$ $\mathrm{D}$ - mannopyranosyl- $(1 \rightarrow 4)$ - 2 - azido - 2-deoxy3,6 - di - O - benzyl - silyl - $\beta$ - D - glucopyranoside (9b). - Compound 9b was synthesized according to the procedure outlined for compound 9a except that disaccharide $\mathbf{7 b}$ was used as the glycosyl donor instead of monosaccharide $7 \mathbf{a}$. After work-up, purification via silica gel chromatography with 2.5:1 hexane-ether provided 9b as an oil ( $36 \%$ yield); $R_{f}$ 9b 0.43 (2.5:1 hexane-ether); ${ }^{1} \mathrm{H} \mathrm{NMR}\left(\mathrm{CDCl}_{3}\right): \delta 0.18$ (s, 3 $\left.\mathrm{H}, \mathrm{CH}_{3}\right), 0.19\left(\mathrm{~s}, 3 \mathrm{H}, \mathrm{CH}_{3}\right), 0.96(\mathrm{~s}, 9 \mathrm{H}$, $t$-butyl), 3.26 (dd, $1 \mathrm{H}, J$ 8.6, $9.9 \mathrm{~Hz}, \mathrm{H}-3$ glucosamine), $3.32-3.40(\mathrm{~m}, 2 \mathrm{H}), 3.54-3.75$ (m), 3.78-3.90 (m, $4 \mathrm{H}), 3.92-4.01(\mathrm{~m}, 2 \mathrm{H})$, $4.20(\mathrm{~d}, 1 \mathrm{H}, J 12.1 \mathrm{~Hz}, H \mathrm{CHPh}), 4.33(\mathrm{~d}, 1$ $\mathrm{H}, J 12.2 \mathrm{~Hz}, H \mathrm{CHPh}), 4.39-4.55$ (m, $14 \mathrm{H})$, $4.61(\mathrm{~d}, 1 \mathrm{H}, J 12.1 \mathrm{~Hz}, H \mathrm{CHPh}), 4.83$ (d, 1 $\mathrm{H}, J 11.0 \mathrm{~Hz}, H \mathrm{CHPh}), 4.86(\mathrm{~d}, 1 \mathrm{H}, J 10.8$ $\mathrm{Hz}, H \mathrm{CHPh}), 4.95$ (d, $1 \mathrm{H}, J 11.5 \mathrm{~Hz}$, $H \mathrm{CHPh}$, 5.07 (d, $1 \mathrm{H}, J 1.03 \mathrm{~Hz}, \mathrm{H}-1$ mannose), 5.26 (d, $1 \mathrm{H}, J 1.8 \mathrm{~Hz}, \mathrm{H}-1$ mannose), 7.09-7.38 (m, phenyl H's); ${ }^{13} \mathrm{C}$ NMR $\left(\mathrm{CDCl}_{3}\right): \delta 99.86(\mathrm{~d}, J 171.5 \mathrm{~Hz}, \mathrm{C}-1$ mannose), 98.20 (d, J $171.3 \mathrm{~Hz}, \mathrm{C}-1$ mannose), 97.15 (d, J $155.6 \mathrm{~Hz}, \mathrm{C}-1$ glucosamine). LRMS (electrospray): Calcd for $\mathrm{C}_{87} \mathrm{H}_{99} \mathrm{~N}_{3}$ $\mathrm{O}_{15} \mathrm{Si}: 1453.7$; Found: 1454.

t-Butyldimethylsilyl 2,3,4,6-tetra-O-benzyl$\alpha$-D-mannopyranosyl- $(1 \rightarrow 2)-3,4,5$-tri-O-ben$z y l-\alpha-\mathrm{D}-$ mannopyranosyl- $(1 \rightarrow 6)-2,3,4-$ tri-Obenzyl- $\alpha$-D-mannopyranosyl- $(1 \rightarrow 4)$ - 2-azido2-deoxy-3,6-di-O-benzyl- $\beta$-D-glucopyranoside (9c). - Compound 9c was synthesized according to the procedure outlined for compound 9a except that trisaccharide 7c was used as the glycosyl donor instead of monosaccharide 7a. After work-up, purification via column chromatography 4:1 hexanes-ether yielded 9c (37\% yield); $R_{f} 9 \mathrm{c} 0.65$ (1:1 hexanes-ether); ${ }^{1} \mathrm{H}$ NMR $\left(\mathrm{CDCl}_{3}\right): \delta 0.15\left(\mathrm{~s}, 6 \mathrm{H}, 2 \mathrm{CH}_{3}\right), 0.96$ (s, $9 \mathrm{H}, t$-butyl), 3.21-3.33 (m, $2 \mathrm{H}), 3.37-3.60$ (m), 3.64-3.92 (m), $4.02(\mathrm{t}, 1 \mathrm{H}, J 9.5 \mathrm{~Hz})$, $4.11(\mathrm{t}, 1 \mathrm{H}, J 2.1 \mathrm{~Hz}), 4.25(\mathrm{~d}, 1 \mathrm{H}, J 12.3 \mathrm{~Hz}$, $H \mathrm{CHPh}), 4.30(\mathrm{~d}, 1 \mathrm{H}, J 12.3 \mathrm{~Hz}, H \mathrm{CHPh})$, 4.37-4.57 (m), 4.58 (d, $1 \mathrm{H}, J 12.3 \mathrm{~Hz}$, $H \mathrm{CHPh}), 4.65$ (d, J $12.3 \mathrm{~Hz}, H \mathrm{CHPh}), 4.79$ (d, $1 \mathrm{H}, J 11.1 \mathrm{~Hz}, H \mathrm{CHPh}), 4.84$ (d, $1 \mathrm{H}, J$ $10.9 \mathrm{~Hz}, H \mathrm{CHPh}), 4.85(\mathrm{~d}, 1 \mathrm{H}, J 11.3 \mathrm{~Hz}$, $H \mathrm{CHPh}), 4.91$ (d, $1 \mathrm{H}, J 11.4 \mathrm{~Hz}, H \mathrm{CHPh})$, 4.92 (d, 1 H, J 1.6 Hz, H-1 mannose), 5.14 (d, $1 \mathrm{H}, J 1.6 \mathrm{~Hz}, \mathrm{H}-1$ mannose), 5.28 (d, $1 \mathrm{H}, J$ $1.9 \mathrm{~Hz}, \mathrm{H}-1$ mannose), 7.12-7.36 (m, phenyl H's); ${ }^{13} \mathrm{C} \mathrm{NMR}\left(\mathrm{CDCl}_{3}\right): \delta 97.12(\mathrm{~d}, J 155 \mathrm{~Hz}$, C-1 glucosamine), 99.27 (d, $J 168.3 \mathrm{~Hz}, 2 \times \mathrm{C}$ 1 mannose), 99.83 (d, J $169.7 \mathrm{~Hz}, \mathrm{C}-1$ mannose).

2,3,4,6-Tetra-O-benzyl- $\alpha$-D-mannopyranosyl$(1 \rightarrow 4)$-2-azido-2-deoxy-3,6-di-O-benzyl- $\alpha, \beta$ D-glucopyranose (29a). — To $360 \mathrm{mg}$ (0.348 $\mathrm{mmol})$ of $9 \mathbf{a}$ in $11 \mathrm{~mL}$ of THF was added 700 $\mu \mathrm{L}$ of glacial $\mathrm{AcOH}$ followed by $5.3 \mathrm{~mL}$ of $t$-butylammonium fluoride (TBAF, $5.32 \mathrm{mmol}$ in THF). The reaction was stirred at $20^{\circ} \mathrm{C}$ for $14 \mathrm{~h}$. The mixture was quenched with $1 \mathrm{~mL}$ of $1 \mathrm{M} \mathrm{NaHCO}_{3}$ and extracted with $\mathrm{CHCl}_{3}(2 \times$ $1 \mathrm{~mL})$. The organic phase was washed sequentially with water $(2 \times 5 \mathrm{~mL})$, satd $\mathrm{NaCl}(5$ $\mathrm{mL})$, dried, and concentrated. Purification via silica gel chromatography yielded $270 \mathrm{mg}$ of 29a (84\% yield); $R_{f}$ 29a 0.29 (1:1 hexanesether); ${ }^{1} \mathrm{H}$ NMR $\left(\mathrm{CDCl}_{3}\right): \delta 2.36-3.40(\mathrm{~m}, 4$ $\mathrm{H}), 3.48-4.05(\mathrm{~m}, 22 \mathrm{H}), 4.21(\mathrm{~d}, 1 \mathrm{H}, J 12.1$ $\mathrm{Hz}), 4.24$ (d, $1 \mathrm{H}, J 12.1 \mathrm{~Hz}), 4.35$ (d, $2 \mathrm{H}, J$ $12.0 \mathrm{~Hz}), 4.41-4.46(\mathrm{~m}, 17 \mathrm{H}), 4.80(\mathrm{~d}, 1 \mathrm{H}, J$ $10.8 \mathrm{~Hz}), 4.82(\mathrm{~d}, 1 \mathrm{H}, J 10.8 \mathrm{~Hz}), 4.90$ (d, 1 $\mathrm{H}, J 10.8 \mathrm{~Hz}), 4.93(\mathrm{~d}, 1 \mathrm{H}, J 10.8 \mathrm{~Hz}), 5.25$ (d, $1 \mathrm{H}, J 2.4 \mathrm{~Hz}, \mathrm{H}-1$ mannose of $\beta$ anomer), $5.27(\mathrm{~d}, 1 \mathrm{H}, J 1.8 \mathrm{~Hz}, \mathrm{H}-1$ mannose of $\alpha$ anomer), 5.33 (d, $1 \mathrm{H}, J 3.4 \mathrm{~Hz}, \mathrm{H}-1$ glucosamine of $\alpha$ anomer), 7.16-7.39 (m, phenyl H's).

2,3,4,6 - Tetra - O - benzyl - $\alpha$ - D - mannopyran osyl- $(1 \rightarrow 6)$ - 2,3,4-tri-O - benzyl- $\alpha$ - D - manno pyranosyl- $(1 \rightarrow 4)$ - 2-azido-2-deoxy-3,6-di-Obenzyl- $\alpha, \beta$-D-glucopyranoside (29b).-Compounds 29b were synthesized according to the 
procedure outlined for compound 29a except that trisaccharide 9b was desilylated instead of disaccharide 9a. After work-up, purification via preparative TLC (1:1 hexanes-ether) yielded 1:1 anomeric mixture of alcohols (85\% yield); $R_{f}$ 29b 0.34 (1:1 hexanes-ether); ${ }^{1} \mathrm{H}$ NMR $\left(\mathrm{CDCl}_{3}\right): \delta 2.43(\mathrm{bs}, 1 \mathrm{H}, \mathrm{OH}), 3.28-$ $3.56(\mathrm{~m}, 4 \mathrm{H}), 3.61-4.02(\mathrm{~m}, 14 \mathrm{H}), 4.16(\mathrm{~d}, 1$ $\mathrm{H}, J 12.1 \mathrm{~Hz}, H \mathrm{CHPh}), 4.31$ (d, $1 \mathrm{H}, J 12 \mathrm{~Hz}$, $H \mathrm{CHPh}), 4.32(\mathrm{~d}, 1 \mathrm{H}, J 12 \mathrm{~Hz}, H \mathrm{CHPh})$, 4.39-4.63 (m, 13.5 H), 4.80-4.91 (m, 3 H), 5.04 (pseudo-s, $1 \mathrm{H}, \mathrm{H}-1$ mannose 2), 5.21 (d, $0.5 \mathrm{H}, J 1.92 \mathrm{~Hz}, \mathrm{H}-1$ mannose 1), 5.23 (d, 0.5 $\mathrm{H}, J 2.01 \mathrm{~Hz}, \mathrm{H}-1$ mannose 1), 5.26 (d, $0.5 \mathrm{H}$, $J 3.32 \mathrm{~Hz}, \mathrm{H}-1$ glucosamine of $\alpha$ anomer), 7.2-7.5 (m, phenyl H's).

2,3,4,6-Tetra-O - benzyl - $\alpha$ - D - mannopyran osyl-(1 $\rightarrow 4)$ - 2-azido-2-deoxy-3,6-di-O-ben$z y l-\alpha, \beta$-D-glucopyranosyl fluoride (10a).Compounds 10a were synthesized according to the procedure outlined for compound $\mathbf{2 2}$ except that 29a was fluorinated instead of $\mathbf{2 1}$. After work-up, the crude products were chromatographed on silica gel with $3: 1$ hexaneether yielding a 1:2 $\alpha, \beta$ mixture of anomeric fluorides ( $84 \%$ yield); $R_{f}$ 10a 0.18 (3:1 hexanes-ether); ${ }^{1} \mathrm{H}$ NMR $\left(\mathrm{CDCl}_{3}\right): \delta$ 3.31$3.41(\mathrm{~m}), 3.42-3.88(\mathrm{~m}), 3.8-4.03(\mathrm{~m}), 4.27(\mathrm{~d}$, $1 \mathrm{H}, J 10.2 \mathrm{~Hz}), 4.31(\mathrm{~d}, 1 \mathrm{H}, J 10.1 \mathrm{~Hz})$, 4.39-4.66 (m), 4.81 (d, $1 \mathrm{H}, J 10.8 \mathrm{~Hz}), 4.83$ (d, $1 \mathrm{H}, J 10.9 \mathrm{~Hz}), 4.87$ (d, $1 \mathrm{H}, J 11.2 \mathrm{~Hz}$ ), $4.88(\mathrm{~d}, 1 \mathrm{H}, J 11.1 \mathrm{~Hz}), 5.09$ (dd, $1 \mathrm{H}, J$ 7.1, $J 52.7 \mathrm{~Hz}, \mathrm{H}-1$ glucosamine of $\beta$ anomer), 5.26 (d, $1 \mathrm{H}, J 2.2 \mathrm{~Hz}, \mathrm{H}-1$ mannose), 5.30 (d, $1 \mathrm{H}, J 2.3 \mathrm{~Hz}, \mathrm{H}-1$ mannose), 5.66 (dd, $1 \mathrm{H}, J$ $2.6, J 52.9 \mathrm{~Hz}, \mathrm{H}-1$ glucosamine of $\alpha$ anomer), 7.14-7.39 (m, phenyl H's).

2,3,4,6-Tetra-O - benzyl- $\alpha$ - D - mannopyranosyl- $(1 \rightarrow 6)$ - 2,3,4-tri-O - benzyl- $\alpha$ - D - manno pyranosyl- $(1 \rightarrow 4)$-2-azido-2-deoxy-3,6-di-Obenzyl- $\alpha, \beta$-D-glucopyranosyl fluoride (10b).Compounds 10b were synthesized according to the procedure outlined for compound $\mathbf{2 2}$ except that 29b was fluorinated instead of $\mathbf{2 1}$. After work-up, purification via preparative TLC (1:1 hexanes-ether) yielded a 1:1 $(\alpha, \beta)$ mixture of fluorides ( $86 \%$ yield); $R_{f} \mathbf{1 0 b} 0.42$ (1:1 hexanes-ether); ${ }^{1} \mathrm{H}$ NMR $\left(\mathrm{CDCl}_{3}\right): \delta$ $3.31-4.02(\mathrm{~m}, 18 \mathrm{H}), 4.24(\mathrm{~d}, 1 \mathrm{H}, J 12.4 \mathrm{~Hz}$, $H \mathrm{CHPh}), 4.25(\mathrm{~d}, 1 \mathrm{H}, 12.1 \mathrm{~Hz}, H \mathrm{CHPh})$, 4.35-4.67 (m, $\left.13 \mathrm{H}, \mathrm{CH}_{2} \mathrm{Ph}\right), 4.81-4.89$ (m, 3
$\mathrm{H}, \mathrm{CH}_{2} \mathrm{Ph}$ ), 5.03 (pseudo-s, $1 \mathrm{H}, \mathrm{H}-1$ mannose 2), $5.07(\mathrm{dd}, 0.5 \mathrm{H}, J 7.1, J 52.7 \mathrm{~Hz}, \mathrm{H}-1$ glucosamine of $\beta$ anomer), $5.22(\mathrm{~d}, 0.5 \mathrm{H}, J$ $1.92 \mathrm{~Hz}, \mathrm{H}-1$ mannose 1), 5.27 (d, $0.5 \mathrm{H}, J 2.1$ Hz, H-1 mannose 1), 5.60 (dd, $0.5 \mathrm{H}, J$ 2.4, J $53 \mathrm{~Hz}, \mathrm{H}-1$ glucosamine of $\alpha$ anomer $), 7.1-7.5$ (m, $45 \mathrm{H}$, phenyl H's).

2,3,4,6-Tetra-O - benzyl - $\alpha$ - D - mannopyranosyl- $(1 \rightarrow 4)$ - 2-azido-2-deoxy-3,6-di-O-benzyl- $\alpha, \beta$-D-glucopyranosyl- $(1 \rightarrow 6)-3,4,5$-tri-Obenzyl-1-D-myo-inositol 1,2-cyclic carbonate $(12 \mathbf{a}, \alpha, \beta)$. - To a pre-dried mixture of donors 10a (45 mg, $0.049 \mathrm{mmol}$ ) and acceptor 11 (47 $\mathrm{mg}, 0.099 \mathrm{mmol})$ was added $4 \AA$ molecular sieves, toluene $(3.7 \mathrm{~mL})$, and $\mathrm{ZrCp}_{2} \mathrm{Cl}_{2}$ (72 $\mathrm{mg}, 0.25 \mathrm{mmol})$. The mixture was cooled to $-42{ }^{\circ} \mathrm{C}$ and AgOTf (127 mg, $0.49 \mathrm{mmol}$ ) was added. The reaction was stirred briefly at $-42{ }^{\circ} \mathrm{C}(\sim 5 \mathrm{~min})$ and then allowed to warm to $20^{\circ} \mathrm{C}$. The reaction was stirred for $5 \mathrm{~h}$, and then quenched by addition of $1 \mathrm{M} \mathrm{NaHCO}$ (6 mL), diluted with $6 \mathrm{~mL}$ of $\mathrm{CH}_{2} \mathrm{Cl}_{2}$, and filtered through Celite. The layers were separated and the aqueous layer was extracted with $\mathrm{CH}_{2} \mathrm{Cl}_{2}(4 \times 4 \mathrm{~mL})$. The combined organic extracts were washed with satd $\mathrm{NaCl}$ (4 $\mathrm{mL}$ ), dried, and concentrated. Purification via preparative TLC (7:3 hexanes-EtOAc) yielded $18.6 \mathrm{mg}$ of $\alpha$ product and $17.8 \mathrm{mg}$ of $\beta$ product (54\% combined yield); $R_{f} \mathbf{1 2 a}, \alpha$ $0.37, R_{f}$ 12a, $\beta 0.45$ (7:3 hexanes-EtOAc); ${ }^{1} \mathrm{H}$ NMR $\left(\mathrm{CDCl}_{3}\right)$ 12a, $\alpha: \delta 3.39(\mathrm{dd}, 1 \mathrm{H}, J$ 3.6, $9.7 \mathrm{~Hz}, \mathrm{H}-2$ glucosamine), 3.51-3.90 (m, 12 $\mathrm{H}$ ), 4.02 (pseudo-t, $2 \mathrm{H}, 9.3 \mathrm{~Hz}$ ), 4.20-4.66 (m, $17 \mathrm{H}), 4.79-5.02(\mathrm{~m}, 4 \mathrm{H}), 5.27$ (d, $1 \mathrm{H}, J$ $1.9 \mathrm{~Hz}, \mathrm{H}-1$ mannose), 5.34 (d, $1 \mathrm{H}, J 3.6 \mathrm{~Hz}$, $\mathrm{H}-1$ glucosamine), 7-7.5 (m, $45 \mathrm{H}$, phenyl H's); ${ }^{1} \mathrm{H}$ NMR $\left(\mathrm{CDCl}_{3}\right)$ 12a, $\beta$ : $\delta 3.23$ (pseudot, $1 \mathrm{H}, J 9.3 \mathrm{~Hz}, \mathrm{H}-2$ glucosamine), 3.37 (m, 1 $\mathrm{H}), 3.44(\mathrm{dd}, 1 \mathrm{H}, J 8.1,9.8 \mathrm{~Hz}), 3.57-3.83$ (m, $10 \mathrm{H}$ ), 3.88 (pseudo-s, $1 \mathrm{H}$ ), 4.00 (pseudo$\mathrm{t}, 1 \mathrm{H}, J 9.2 \mathrm{~Hz}), 4.30(\mathrm{~m}, 2 \mathrm{H}), 4.37-4.63(\mathrm{~m}$, $14 \mathrm{H}), 4.70$ (d, $1 \mathrm{H}, J 10.6 \mathrm{~Hz}, H \mathrm{CHPh}), 4.82$ (d, $1 \mathrm{H}, J 10 \mathrm{~Hz}, H \mathrm{CHPh}$ ), 4.82 (pseudo-s, 2 $\left.\mathrm{H}, \mathrm{CH}_{2} \mathrm{Ph}\right), 4.88$ (d, $\left.1 \mathrm{H}, J 11.3 \mathrm{~Hz}, H \mathrm{CHPh}\right)$, $5.31(\mathrm{~d}, 1 \mathrm{H}, J 2.0 \mathrm{~Hz}, \mathrm{H}-1$ mannose), 7-7.5 (m, $45 \mathrm{H}$, phenyl H's). LRMS 12a, $\alpha$ : Calcd for $\mathrm{C}_{82} \mathrm{H}_{83} \mathrm{~N}_{3} \mathrm{O}_{16}+\mathrm{Na}$ : 1388.57; Found: 1388 .

2,3,4,6-Tetra-O-benzyl- $\alpha$-D-mannopyranosyl$(1 \rightarrow 6)-2,3,4$ - tri-O-benzyl- $\alpha$ - D-mannopyranosyl- $(1 \rightarrow 4)$ - 2-azido-2-deoxy-3,6-di-O - benzyl - $\alpha, \beta$ - D - glucopyranosyl - $(1 \rightarrow 6)$ - 3,4,5 - tri- 
O-benzyl-1-D-myo-inositol 1,2 cyclic carbonate $(\mathbf{1 2 b}, \alpha, \beta)$. Compounds $12 \mathbf{b}, \alpha, \beta$ were synthesized in a manner analogous to that for compounds 12a, $\alpha, \beta$ except that trisaccharides $\mathbf{1 0 b}$ were used as glycosyl donor instead of disaccharides 10a. After work-up, purification via preparative TLC (7:3 hexanes-EtOAc) yielded $17.5 \mathrm{mg}$ of $\mathbf{1 2 b}, \alpha$ and $6.6 \mathrm{mg}$ of slightly impure 12b, $\beta$ (57\% combined yield); $R_{f} \mathbf{1 2 b}, \alpha$ 0.38, $R_{f} \mathbf{1 2 b}, \beta \quad 0.46 \quad(7: 3$ hexanesEtOAc); ${ }^{1} \mathrm{H}$ NMR $\left(\mathrm{CDCl}_{3}\right)$ 12b, $\alpha: \delta 3.37(\mathrm{dd}$, $1 \mathrm{H}, J 3.6,9.6 \mathrm{~Hz}, \mathrm{H}-2$ glucosamine), 3.49-3.7 $(\mathrm{m}, 11 \mathrm{H}), 3.75-3.88(\mathrm{~m}, 8 \mathrm{H}), 3.94-4.0(\mathrm{~m}, 3$ $\mathrm{H}), 4.16(\mathrm{~d}, 1 \mathrm{H}, J 12.0 \mathrm{~Hz}, H \mathrm{CHPh}), 4.27-$ $4.65(\mathrm{~m}, 19 \mathrm{H}), 4.80-4.91(\mathrm{~m}, 5 \mathrm{H}), 5.04$ (pseudo-s, $1 \mathrm{H}, \mathrm{H}-1$ mannose), 5.23 (pseudo-s, $1 \mathrm{H}, \mathrm{H}-1$ mannose), $5.32(\mathrm{~d}, 1 \mathrm{H}, J 3.6 \mathrm{~Hz}$, $\mathrm{H}-1$ glucosamine $), 7.0-7.5(\mathrm{~m}, 60 \mathrm{H}$, phenyl H's); ${ }^{13} \mathrm{C} \quad \mathrm{NMR} \quad\left(\mathrm{CDCl}_{3}\right)$ 12b, $\alpha: \quad \delta \quad 96.21$ (anomeric C), 98.10 (anomeric C), 100.40 (anomeric C), 153.97 (carbonate carbon); LRMS: Calcd for $\mathrm{C}_{109} \mathrm{H}_{111} \mathrm{~N}_{3} \mathrm{O}_{21}+\mathrm{NH}_{4}$ 1815.8; Found: 1816.

2,3,4,6-Tetra-O - benzyl - $\alpha$ - D - mannopyran osyl- $(1 \rightarrow 2)-3,4,6$ - tri-O - benzyl - $\alpha$ - D - manno pyranosyl - $(1 \rightarrow 6)-2,3,4-$ tri - O - benzyl $-\alpha-\mathrm{D}-$ mannopyranosyl-(1 $\rightarrow 4)$-2-azido-2-deoxy-3,6di - O - benzyl - $\alpha, \beta$ - D - glucopyranosyl - $(1 \rightarrow 6)$ 3,4,5-tri-O-benzyl-1-D-myo-inositol 1,2-cyclic carbonate (12c, $\alpha, \beta)$.-Compounds 12c, $\alpha, \beta$ were synthesized in a manner analogous to that for compounds $\mathbf{1 2} \mathbf{a}, \alpha, \beta$ except that tetrasaccharides 10c were used as the glycosyl donor instead of disaccharides 10a. After work-up, purification via preparative TLC (7:3 hexanes-EtOAc) yielded $5.2 \mathrm{mg}$ of $12 \mathrm{c}, \alpha$ and $2.0 \mathrm{mg}$ of $12 \mathrm{c}, \beta$ (53\% combined yield); $R_{f}$ $12 \mathrm{c}, \alpha \quad 0.44, \quad R_{f} \quad 12 \mathrm{c}, \beta \quad 0.52 \quad(7: 3$ hexanesEtOAc); ${ }^{1} \mathrm{H}$ NMR $\left(\mathrm{CDCl}_{3}\right)$ 12c, $\alpha: \delta 3.28(\mathrm{dd}$, $1 \mathrm{H}, J 3.5, J 9.7 \mathrm{~Hz}, \mathrm{H}-2$ glucosamine), $3.38-$ $3.94(\mathrm{~m}, 24 \mathrm{H}), 3.98-4.09(\mathrm{~m}, 3 \mathrm{H}), 4.23-4.67$ $(\mathrm{m}, 27 \mathrm{H}), 4.76-4.89(\mathrm{~m}, 6 \mathrm{H}), 5.13(\mathrm{~s}, 1 \mathrm{H}$, $\mathrm{H}-1$ mannose), 5.27 (m, $2 \mathrm{H}, \mathrm{H}-1$ mannose, $\mathrm{H}-1$ glucosamine $)$, 7.2-7.4 (m, $75 \mathrm{H}$, phenyl H's).

2,3,4,6-Tetra - O - benzyl - $\alpha$ - D - mannopyran osyl-(1 $\rightarrow 4)$ - 2-azido-2-deoxy-3,6-di-O-ben$z y l-\alpha-\mathrm{D}$ - glucopyranosyl - $(1 \rightarrow 6)$ - 3,4,5 - triO-benzyl-1-D-myo-inositol (30a, $\alpha)$. - To 17.5 $\mathrm{mg}$ of 12a, $\alpha(0.0128 \mathrm{mmol})$ in $1.4 \mathrm{~mL}$ of THF was added $192 \mu \mathrm{L}$ of $1 \mathrm{M} \mathrm{LiOH}$. The reaction was stirred at $20^{\circ} \mathrm{C}$ for $17 \mathrm{~h}$ and quenched by addition of $1 \mathrm{M} \mathrm{NH}_{4} \mathrm{Cl}(3 \mathrm{~mL})$. The mixture was extracted with $\mathrm{CH}_{2} \mathrm{Cl}_{2}(4 \times 3 \mathrm{~mL})$. The combined organic extracts were washed with satd $\mathrm{NaCl}(3 \mathrm{~mL})$, dried, and concentrated. Purification via preparative TLC $\left(1: 1 \mathrm{CHCl}_{3}-\right.$ ether) yielded $13.8 \mathrm{mg}$ of diol $\mathbf{3 0 a}, \alpha \quad(80 \%$ yield); $R_{f}$ 30a, $\alpha$ o.57 (1:1 $\mathrm{CHCl}_{3}$-ether); ${ }^{1} \mathrm{H}$ NMR $\left(\mathrm{CDCl}_{3}\right): \delta 2.51$ (bs, OH), 3.29-3.56 (m), 3.58-3.63 (m), 3.65-4.06 (m), 4.16 (pseudo-t, $1 \mathrm{H}, J 2.7 \mathrm{~Hz}, \mathrm{H}-2$ inositol), 4.23 (d, $1 \mathrm{H}, J 12.2 \mathrm{~Hz}, H \mathrm{CHPh}), 4.26-4.38(\mathrm{~m})$, 4.44-4.68 (m), 4.72-4.94 (m), 5.23 (d, $1 \mathrm{H}, J$ $2.1 \mathrm{~Hz}, \mathrm{H}-1$ mannose), 5.45 (d, $1 \mathrm{H}, J 3.6 \mathrm{~Hz}$, H-1 glucosamine), 7.10-7.39 (m, phenyl H's).

2,3,4,6-Tetra-O - benzyl - $\alpha$ - D - mannopyranosyl-(1 $\rightarrow 4)$-2-azido-2-deoxy-3,6-di-O-benzyl- $\beta$ - D-glucopyranosyl- $(1 \rightarrow 6)$ - 3,4,5-tri- Obenzyl-1-D-myo-inositol (30a, $\beta)$. - Compound 30a, $\beta$ was synthesized in a manner analogous to that for compound 30a, $\alpha$ except that $12 \mathbf{a}, \beta$ was hydrolyzed instead of $\mathbf{1 2 a}, \alpha$. After workup, purification via preparative TLC (1:1 $\mathrm{CHCl}_{3}$-ether) yielded $12.3 \mathrm{mg}$ of diol 30a, $\beta$ (75\% yield); $R_{f}$ 30a, $\beta$ o.80 (1:1 $\mathrm{CHCl}_{3}$-ether); ${ }^{1} \mathrm{H} \mathrm{NMR}\left(\mathrm{CDCl}_{3}\right): \delta 3.26$ (pseudo-t, $1 \mathrm{H}, J 10$ $\mathrm{Hz}, \mathrm{H}-2$ glucosamine), 3.4-4.1 (m, $15 \mathrm{H}), 4.2$ (pseudo-t, $1 \mathrm{H}, J$ 2.7, H-2 inositol), 4.25-5.1 (m, $20 \mathrm{H}), 5.26$ (pseudo-s, $1 \mathrm{H}, \mathrm{H}-1$ mannose), $7.0-7.5$ (m, $45 \mathrm{H}$, phenyl H's).

2,3,4,6 - Tetra - O - benzyl - $\alpha$ - D - mannopyran osyl- $(1 \rightarrow 6)$ - 2,3,4-tri-O - benzyl- $\alpha$ - D - manno pyranosyl- $(1 \rightarrow 4)$-2-azido-2-deoxy-3,6-di-Obenzyl- $\alpha$-D - glucopyranosyl- $(1 \rightarrow 6)-3,4,5$ - triO-benzyl-1-D-myo-inositol (30b, $\alpha)$. - Compound 30b, $\alpha$ was synthesized in a manner analogous to that for compound 30a, $\alpha$ except that $12 \mathbf{b}, \alpha$ was hydrolyzed instead of $12 \mathbf{a}, \alpha$. After work-up, purification via preparative TLC (1:1 $\mathrm{CHCl}_{3}$-ether) yielded $14 \mathrm{mg}$ of diol (86\% yield); $R_{f}$ 30b, $\alpha 0.61$ (1:1 $\mathrm{CHCl}_{3}$-ether); ${ }^{1} \mathrm{H} \mathrm{NMR}\left(\mathrm{CDCl}_{3}\right) \delta 2.6(\mathrm{~s}, 1 \mathrm{H}, \mathrm{OH}), 3.25-$ $3.66(\mathrm{~m}, 11 \mathrm{H}), 3.71$ (pseudo-t, $1 \mathrm{H}, J 2.3 \mathrm{~Hz}$ ), 3.79-3.85 (m, 6 H), 3.87-4.02 (m, $5 \mathrm{H}), 4.14$ $4.18(\mathrm{~m}, 2 \mathrm{H}), 4.21-4.50(\mathrm{~m}, 12 \mathrm{H}), 4.54(\mathrm{~d}, 1$ $\mathrm{H}, J 11.9 \mathrm{~Hz}, H \mathrm{CHPh}), 4.58(\mathrm{~d}, 1 \mathrm{H}, J 12.0$ $\mathrm{Hz}, H \mathrm{CHPh}), 4.64(\mathrm{~d}, 1 \mathrm{H}, J 11.3 \mathrm{~Hz}$, $H \mathrm{CHPh}), 4.70-4.76(\mathrm{~m}, 4 \mathrm{H}), 4.82-4.90$ (m, 4 $\mathrm{H}), 4.94(\mathrm{~d}, 1 \mathrm{H}, J 11.0 \mathrm{~Hz}, H \mathrm{CHPh}), 5.03$ (pseudo-s, $1 \mathrm{H}, \mathrm{H}-1$ mannose), 5.22 (d, $1 \mathrm{H}, J$ $1.7 \mathrm{~Hz}, \mathrm{H}-1$ mannose), 5.44 (d, $1 \mathrm{H}, J 3.6 \mathrm{~Hz}$, 
H-1 glucosamine), 7.2-7.5 (m, $60 \mathrm{H}$, phenyl H's).

2,3,4,6-Tetra-O-benzyl- $\alpha$-D-mannopyranosyl$(1 \rightarrow 6)$ - 2,3,4-tri-O-benzyl- $\alpha$ - D-mannopyranosyl-(1 $\rightarrow 4)$-2-azido-2-deoxy-3,6-di-O - benzyl- $\beta$ - D - glucopyranosyl- $(1 \rightarrow 6)$ - 3,4,5-tri-Obenzyl-1-D-myo-inositol $(\mathbf{3 0 b}, \beta)$. - Compound 30b, $\beta$ was synthesized in a manner analogous to that for compound $\mathbf{3 0 a}, \alpha$ except that $\mathbf{1 2 b}, \beta$ was hydrolyzed instead of $\mathbf{1 2 a}, \alpha$. After workup, purification via preparative TLC (1:1 $\mathrm{CHCl}_{3}$-ether) yielded $4.3 \mathrm{mg}$ of diol $(66 \%$ yield); ${ }^{1} \mathrm{H}$ NMR $\left(\mathrm{CDCl}_{3}\right): \delta 2.50(\mathrm{bs}, 1 \mathrm{H}$, $\mathrm{OH}$ ), 3.28 (pseudo-t, $1 \mathrm{H}, J 9.3 \mathrm{~Hz}$ ), 3.35-4.07 $(\mathrm{m}, 26 \mathrm{H}), 4.17-4.22(\mathrm{~m}, 2 \mathrm{H}), 4.32-4.62$ (m, $13 \mathrm{H}), 4.68-5.03$ (m, $9 \mathrm{H}), 5.24$ (pseudo-s, 1 $\mathrm{H}, \mathrm{H}-1$ mannose $), 7.0-7.4(\mathrm{~m}, 60 \mathrm{H}$, phenyl Hs).

2,3,4,6 - Tetra - O - benzyl - $\alpha$ - D - mannopyran osyl- $(1 \rightarrow 2)-3,4,6$ - tri- O - benzyl - $\alpha$ - D - manno pyranosyl - $(1 \rightarrow 6)-2,3,4-$ tri - O - benzyl - $\alpha-\mathrm{D}-$ mannopyranosyl-(1 $\rightarrow 4)$-2-azido-2-deoxy-3,6di - O - benzyl - $\alpha$ - D - glucopyranosyl - $(1 \rightarrow 6)$ 3,4,5-tri-O-benzyl-1-D-myo-inositol $(\mathbf{3 0 c}, \alpha)$.Compound 30c, $\alpha$ was synthesized in a manner analogous to that for compound 30a, $\alpha$ except that $12 \mathbf{c}, \alpha$ was hydrolyzed instead of $12 \mathbf{a}, \alpha$. After work-up, purification via preparative TLC (1:1 $\mathrm{CHCl}_{3}$-ether) yielded $5.1 \mathrm{mg}$ of diol 30c, $\alpha\left(97 \%\right.$ yield); ${ }^{1} \mathrm{H}$ NMR $\left(\mathrm{CDCl}_{3}\right): \delta 2.50$ (bs, $1 \mathrm{H}, \mathrm{OH}), 3.28-4.95(\mathrm{~m}, 60 \mathrm{H}), 5.02$ (pseudo-s, $1 \mathrm{H}, \mathrm{H}-1$ mannose), 5.14 (pseudo-s, $1 \mathrm{H}, \mathrm{H}-1$ mannose), 5.25 (pseudo-s, $1 \mathrm{H}, \mathrm{H}-1$ mannose), 5.40 (pseudo-s, $1 \mathrm{H}, \mathrm{H}-1$ glucosamine) 7.0-7.4 (m, $75 \mathrm{H}$, phenyl H's).

Preparation of phosphorylating reagent $31^{18}$ - Methyl dichlorophosphoridate $\left(\mathrm{PCl}_{2}-\right.$ $\mathrm{O}_{2} \mathrm{Me}, 500 \mu \mathrm{L}$ ) was added slowly to $5.0 \mathrm{~mL}$ of freshly-distilled pyridine. The reaction was stirred at $20^{\circ} \mathrm{C}$ for $30 \mathrm{~min}$ prior to use.

$\alpha$-D-Mannopyranosyl-(1 $\rightarrow 4)$ - 2-amino-2-deoxy - $\alpha$ - D - glucopyranosyl - $(1 \rightarrow 6)$ - 1 - D - myoinositol 1:2-(cyclic) phosphate (1). - To a solution of 30a, $\alpha(13.0 \mathrm{mg}, 9.70 \mu \mathrm{mol})$ in pyridine $(230 \mu \mathrm{L})$ was added freshly prepared phosphorylating reagent $\mathbf{3 1}(518 \mu \mathrm{L})$. The reaction was stirred at $20^{\circ} \mathrm{C}$ until judged complete $(30 \mathrm{~min})$ by TLC analysis $\left(1: 1: 1, \mathrm{CHCl}_{3}-\right.$ ether- $\mathrm{MeOH}$, $R_{f}$ diol $1.0, R_{f}$ cyclic phosphate $0.73, R_{f}$ acyclic $0 ; 1: 1, \mathrm{CHCl}_{3}$-ether, $R_{f}$ diol $0.57, R_{f}$ cyclic and acyclic phosphate 0 ). The reaction was quenched by addition of $1 \mathrm{~mL}$ of satd $\mathrm{NaHCO}_{3}$ and concentrated by coevaporation with heptane. The solid was dissolved in $3 \mathrm{~mL}$ of water followed by dropwise acidification with $2 \mathrm{M} \mathrm{HCl}$ to $\mathrm{pH} 1$ ( $\sim 20$ drops). The suspension was extracted with EtOAc $(5 \times 3$ $\mathrm{mL})$ and the combined organic extracts were dried $\left(\mathrm{Na}_{2} \mathrm{SO}_{4}\right)$, concentrated, dried by coevaporation with toluene, and dissolved in dry THF (2.5 mL). In a separate flask, $\mathrm{NH}_{3}(5$ $\mathrm{mL})$ was condensed at $-78^{\circ} \mathrm{C}$ and $\mathrm{Na}(45$ $\mathrm{mg}, 1.94 \mathrm{mmol}$ ) was added. Once the blue color persisted, the THF solution was added dropwise. The reaction mixture was stirred at $-78^{\circ} \mathrm{C}$ for $15 \mathrm{~min}$ (blue color persisted) and then carefully quenched by addition of $167 \mathrm{mg}$ (3.12 mmol) of $\mathrm{NH}_{4} \mathrm{Cl}$ (s) at $-78^{\circ} \mathrm{C}$. The suspension was stirred vigorously at $-78^{\circ} \mathrm{C}$ until the blue color had completely disappeared. Methanol $(6.0 \mathrm{~mL})$ was added at $-78{ }^{\circ} \mathrm{C}$, the cooling bath and the septum covering the flask were removed, and the reaction mixture was allowed to thaw and evaporate for $12 \mathrm{~h}$ at $20^{\circ} \mathrm{C}$. The resulting white powder was dissolved in water $(4 \mathrm{~mL})$ and filtered through Celite. The filtrate was evaporated to dryness, dissolved in water $(1.0 \mathrm{~mL})$, and desalted by passing over $12 \mathrm{~g}$ of Sephadex G-10, eluting with water $(12 \mathrm{~mL})$. Evaporation of the eluate yielded $3.1 \mathrm{mg}$ of $1(56 \%$ yield over two steps). ${ }^{1} \mathrm{H}$ NMR $\left(\mathrm{D}_{2} \mathrm{O}\right): \delta 3-4$ (m, $16 \mathrm{H}), 4.35$ (ddd, $1 \mathrm{H}, \mathrm{H}-1$ inositol), 4.5 (pseudo-t, $1 \mathrm{H}, \mathrm{H}-2$ inositol), 5.1 (pseudo-s, 1 $\mathrm{H}, \mathrm{H}-1$ mannose), 5.3 (pseudo-s, $1 \mathrm{H}, \mathrm{H}-1$ glucosamine); ${ }^{31} \mathrm{P}$ NMR $\left(\mathrm{D}_{2} \mathrm{O}\right): \delta 3.62$ (trace of acyclic phosphate), 17.14 (cyclic phosphate); HRMS (FAB, negative ion mode): Calcd for $\mathrm{C}_{18} \mathrm{H}_{31} \mathrm{NO}_{17} \mathrm{P}$ : 564.1329; Found: 564.1354.

$\alpha$ - D - Mannopyranosyl - $(1 \rightarrow 4)$ - 2 - amino - 2deoxy- $\beta$ - D-glucopyranosyl- $(1 \rightarrow 6)$ - 1 - D-myoinositol 1:2-(cyclic) phosphate (2).-Compound $\mathbf{2}$ was synthesized in a manner analogous to that for compound 1 except that $\beta$ anomer 30a, $\beta$ was phosphorylated and deprotected instead of the $\alpha$ anomer 30a, $\alpha$. After work-up, desalting with Sephadex G-10 yielded $8.0 \mathrm{mg}$ of $\mathbf{2}$ (84\% yield over two steps); ${ }^{1} \mathrm{H}$ NMR $\left(\mathrm{D}_{2} \mathrm{O}\right): \delta 2.9(\mathrm{~m}, 1 \mathrm{H}), 3.3-4(\mathrm{~m}, 15$ $\mathrm{H}), 4.4$ (ddd, $1 \mathrm{H}, \mathrm{H}-1$ inositol), 4.55 (pseudos, $1 \mathrm{H}, \mathrm{H}-2$ inositol), 4.85 (pseudo-s, $1 \mathrm{H}, \mathrm{H}-1$ 
glucosamine), 5.1 (pseudo-s, $1 \mathrm{H}, \mathrm{H}-1$ mannose); ${ }^{31} \mathrm{P}$ NMR $\left(\mathrm{D}_{2} \mathrm{O}\right): \delta 16.79$.

$\alpha$-D - Mannopyranosyl- $(1 \rightarrow 6)-\alpha-\mathrm{D}-$ mannopyranosyl- $(1 \rightarrow 4)$-2-amino-2-deoxy- $\alpha$-D-glucopyranosyl-(1 $\rightarrow 6)-1$-D-myo-inositol 1:2(cyclic) phosphate (3). - Compound 3 was synthesized in a manner analogous to that for compound 1 except that tetrasaccharide $\mathbf{3 0 b}, \alpha$ was phosphorylated and deprotected instead of trisaccharide 30a, $\alpha$. After work-up, desalting with Sephadex G-10 yielded $3.1 \mathrm{mg}$ of 3 (75\% yield over two steps). ${ }^{1} \mathrm{H}$ NMR $\left(\mathrm{D}_{2} \mathrm{O}\right): \delta$ $3.1-4.0(\mathrm{~m}, 22 \mathrm{H}), 4.35$ (ddd, $1 \mathrm{H}, J$ 4, 12, 20 $\mathrm{Hz}, \mathrm{H}-1$ inositol), 4.5 (pseudo-t, $1 \mathrm{H}, J 4 \mathrm{~Hz}$, $\mathrm{H}-2$ inositol), 4.7 (pseudo-s, $1 \mathrm{H}, \mathrm{H}-1$ mannose), 5.05 (pseudo-s, $1 \mathrm{H}, \mathrm{H}-1$ mannose), 5.33 (d, $1 \mathrm{H}, \mathrm{H}-1$ glucosamine); ${ }^{31} \mathrm{P}$ NMR $\left(\mathrm{D}_{2} \mathrm{O}\right): \delta$ 17.13; HRMS (electrospray): Calcd for $\left[\mathrm{C}_{24} \mathrm{H}_{42} \mathrm{NO}_{22} \mathrm{P}+\mathrm{H}\right]^{+}$728.2014; Found: 728.1996.

$\alpha$ - D - Mannopyranosyl- $(1 \rightarrow 6)-\alpha-\mathrm{D}-$ manno pyranosyl- $(1 \rightarrow 4)$ - 2-amino-2-deoxy- $\beta$ - D-glucopyranosyl - $(1 \rightarrow 6)$ - 1 - D - myo - inositol 1:2(cyclic) phosphate (4). - Compound 4 was synthesized in a manner analogous to that for compound 1 except that $\beta$-tetrasaccharide $\mathbf{3 0 b}, \beta$ was phosphorylated and deprotected instead of $\alpha$-trisaccharide 30a, $\alpha$. After work-up, desalting with Sephadex G-10 yielded $3.1 \mathrm{mg}$ of 4 (83\% yield over two steps). ${ }^{1} \mathrm{H}$ NMR $\left(\mathrm{D}_{2} \mathrm{O}\right): \delta 2.97(\mathrm{~m}, 1 \mathrm{H}), 3.33$ (pseudo-t, $\left.1 \mathrm{H}\right)$, 3.44-4.14 (m, $20 \mathrm{H}), 4.29$ (ddd, $1 \mathrm{H}, J 4.7$, 8.1, $20 \mathrm{~Hz}, \mathrm{H}-1$ inositol), 4.5 (pseudo-t, $1 \mathrm{H}$, $\mathrm{H}-2$ inositol), 4.73 (pseudo-s, $1 \mathrm{H}, \mathrm{H}-1$ mannose), 4.81 (d, $1 \mathrm{H}, J 7.4 \mathrm{~Hz}, \mathrm{H}-1$ glucosamine), 5.03 (pseudo-s, $1 \mathrm{H}, \mathrm{H}-1$ mannose); ${ }^{31} \mathrm{P}$ NMR $\left(\mathrm{D}_{2} \mathrm{O}\right): \delta 16.89$.

$\alpha$-D - Mannopyranosyl- $(1 \rightarrow 2)-\alpha-\mathrm{D}$ - manno pyranosyl- $(1 \rightarrow 6)-\alpha$-D-mannopyranosyl- $(1 \rightarrow 4)$ 2 -amino-2-deoxy- $\alpha$-D-glucopyranosyl- $(1 \rightarrow 6)$ 1-D-myo-inositol 1:2-(cyclic) phosphate (5).Compound $\mathbf{5}$ was synthesized in a manner analogous to that for compound $\mathbf{1}$ except that $\alpha$-pentasaccharide $30 \mathbf{c}, \alpha$ was phosphorylated and deprotected instead of $\alpha$-trisaccharide 30a, $\alpha$. After work-up, desalting with Sephadex G-10 yielded $1.1 \mathrm{mg}$ of 5 (75\% yield over two steps); ${ }^{1} \mathrm{H}$ NMR $\left(\mathrm{D}_{2} \mathrm{O}\right): \delta 2.62$ (pseudo-d, $1 \mathrm{H}$, $J 7.7 \mathrm{~Hz}, \mathrm{H}-2$ glucosamine), 3.20 (pseudo-t, 1 $\mathrm{H}, J 9.5 \mathrm{~Hz}$ ), 3.39-3.88 (m, $26 \mathrm{H}), 4.36$ (ddd, $1 \mathrm{H}, \mathrm{H}-1$ inositol), 4.5 (pseudo-t, $1 \mathrm{H}, \mathrm{H}-2$ inositol), 4.84 (pseudo-s, $1 \mathrm{H}, \mathrm{H}-1$ mannose), 4.95 (pseudo-s, $1 \mathrm{H}, \mathrm{H}-1$ mannose), 5.04 (pseudo-s, $1 \mathrm{H}, \mathrm{H}-1$ mannose), 5.13 (d, $1 \mathrm{H}, J$ $3.1 \mathrm{~Hz}, \mathrm{H}-1$ glucosamine); ${ }^{31} \mathrm{P}$ NMR $\left(\mathrm{D}_{2} \mathrm{O}\right): \delta$ 17.12; HRMS (FAB, negative ion mode): Calcd for $\mathrm{C}_{30} \mathrm{H}_{51} \mathrm{NO}_{27} \mathrm{P}$ : 888.2385; Found: 888.2415 .

\section{Acknowledgements}

We are grateful to the National Institutes of Health (grant no. DK44589) for financial support.

\section{References}

1. National Institutes of Health. Diabetes in America, 2nd ed.; NIH Publication, 1995.

2. Jones, D. R.; Varela-Nieto, I. Int. J. Biochem. Cell Biol. 1998, 30, 313-326.

3. Misek, D. E.; Saltiel, A. R. Endocrinology 1994, 135, 1869-1876.

4. Asplin, I.; Galasko, G.; Larner, J. Proc. Natl. Acad. Sci. USA 1993, 90, 5924-5928.

5. Mato, J. M.; Kelly, K.; Abler, L.; Jarrett, L.; Corkey, B. E.; Cashel, B. E.; Zopf, D. Biochem. Biophys. Res. Commun. 1988, 146, 764-770.

6. Larner, J.; Huang, L. C.; Schwartz, C. F. W.; Oswald, A. S.; Shen, T.-Y.; Kinter, M.; Tang, G.; Zeller, K. Biochem. Biophys. Res. Commun. 1988, 151, 1416-1426.

7. Reddy, K. K.; Falck, J. R.; Capdevila, J. Tetrahedron Lett. 1993, 34, 7869-7872.

8. Frick, W.; Bauer, A.; Bauer, J.; Wied, S.; Müller, G. Biochemistry 1998, 37, 13421-13436.

9. Garegg, P. J.; Konradsson, P.; Lezdins, D.; Oscarson, S.; Ruda, K.; Ohberg, L. Pure Appl. Chem. 1998, 70, 293298.

10. Dietrich, H.; Espinosa, J. F.; Chiara, J. L.; Jimenez-Barbero, J.; Leon, Y.; Varela-Nieto, I.; Mato, J.-M.; Cano, F. H.; Foces-Foces, C.; Martin-Lomas, M. Chem. Eur. J. 1999, 5, 320-336.

11. Jaworek, C. H.; Calias, P.; Iacobucci, S.; d'Alarcao, M. Tetrahedron Lett. 1999, 40, 667-670.

12. See Refs. 10 and 11 .

13. Martin-Lomas, M.; Khiar, N.; Garcia, S.; Koessler, J.-L.; Nieto, P. M.; Rademacher, T. W. Chem. Eur. J. 2000, 6, 2608-2621.

14. Misek, D. E.; Saltiel, A. R. J. Biol. Chem. 1992, 267, $16266-16273$.

15. Murakata, C.; Ogawa, T. Carbohydr. Res. 1992, 234, 75-91

16. Yamazaki, F.; Sato, S.; Nukada, T.; Ito, Y.; Ogawa, T. Carbohydr. Res. 1990, 201, 31-50.

17. Matsumoto, T.; Maeta, H.; Suzuki, K.; Tsuchihashi, G. Tetrahedron Lett. 1988, 29, 3567-3570.

18. Smrt, J.; Catlin, J. Tetrahedron Lett. 1970, 58, 50815082.

19. Mori, M.; Ito, Y.; Ogawa, T. Carbohydr. Res. 1989, 192, $131-146$ 
20. Massiot, G.; Lavaud, C.; Besson, V. Bull. Soc. Chim. Fr. 1990, 127, 440-445.

21. Bleasdale, C.; Ellwood, S. B.; Golding, B. T. J. Chem. Soc., Perkin Trans. 1 1990, 803-805.

22. Ito, Y.; Ogawa, T. Tetrahedron Lett. 1988, 29, 1061-1064.

23. Boch, K.; Pedersen, C. J. Chem. Soc., Perkin Trans. 21974, 293-299.

24. Iacobucci, S.; Filippova, N.; d'Alarcao, M. Carbohydr.
Res. 1995, 277, 321-325.

25. Posner, G. H.; Haines, S. R. Tetrahedron Lett. 1985, 26, 5-8.

26. Peach, M. E. Can. J. Chem. 1968, 46, 211-215.

27. Kornienko, A.; Turner, D. I.; Jaworek, C. H.; d'Alarcao, M. Tetrahedron: Asymmetry 1998, 9, 2783-2786.

28. Kirchner, J. G. Thin Layer Chromatography, 2nd ed.; Wiley: New York, 1978; Vol. XIV. 